\title{
Neural Synchrony for Adaptive Control
}

\author{
Pieter Verbeke and Tom Verguts
}

\begin{abstract}
Cognitive control can be adaptive along several dimensions, including intensity (how intensely do control signals influence bottom-up processing) and selectivity (what information is selected for further processing). Furthermore, control can be exerted along slow or fast time scales. Whereas control on a slow time scale is used to proactively prepare for upcoming challenges, control can also be used on a faster time scale to react to unexpected events that require control. Importantly, a systematic comparison of these dimensions and time scales remains lacking. Moreover, most current models of adaptive control allow
\end{abstract}

\section{INTRODUCTION}

While driving a car, it is important to focus on the road ahead and not on any interesting events that might be happening on the sidewalk. This ability to efficiently select and process relevant information is generally referred to as cognitive control (e.g., Norman \& Shallice, 1986). According to theoretical work (e.g., Verguts \& Notebaert, 2008; Botvinick, Braver, Barch, Carter, \& Cohen, 2001; Cohen, Dunbar, \& McClelland, 1990), cognitive control is implemented by a top-down biasing signal that excites neural processing pathways carrying relevant information.

Importantly, human cognitive control is also highly adaptive. For instance, a driver's focus on the road might be more intense when driving in a big city during rush hour than when it is the only car on a deserted highway. This adaptive nature of control is also observed in experimental settings. The most well-known illustration of adaptive control is probably the conflict-adaptation effect (Gratton, Coles, \& Donchin, 1992). This effect can be observed in congruency tasks where a stimulus consists of two or more (conflicting) features. For example, in a Stroop task, on each trial, a color word (e.g., "blue") is presented in a particular font color (e.g., red). The participant then has to respond to one feature of the stimulus (font color) and ignore the other feature (word). If all features of the stimulus provide evidence for the same response (e.g., "blue" presented in blue), then this trial is considered congruent; when the stimulus features provide conflicting evidence (e.g., "blue" presented in red), this is considered an incongruent trial. Participants perform

Ghent University predictions only at a behavioral, not neurophysiological, level, thus seriously reducing the range of available empirical restrictions for informing model formulation. The current article addresses this issue by implementing a control loop in an earlier model of neural synchrony. The resulting model is tested on a Stroop task. We observe that only the model that exerts cognitive control on intensity and selectivity dimensions, as well as on two time scales, can account for relevant behavioral and neurophysiological data. Our findings hold important implications for both cognitive control and how computational models can be empirically constrained.

better (faster and more accurate responses) on congruent trials than on incongruent trials. The conflict-adaptation effect entails that performance depends not only on the congruency of the current trial $(n)$ but also on the congruency of the previous trial $(n-1)$. Specifically, performance improves when the congruency of the current trial matches the congruency of the previous trial (i.e., interaction between congruency on trial $n$ and trial $n-1$ ).

Usually, the conflict-adaptation effect is explained by the hypothesis that participants increase the "intensity" of control after incongruent trials. More generally, an emerging consensus suggests that the brain computes a quantity that evaluates the required intensity of control. The original instantiation of this idea proposed response conflict as the quantity that an agent may compute for that purpose (Botvinick et al., 2001). Under this explanation, incongruent trials evoke considerable response conflict, which results in an increase of control intensity and an increased performance on the next (incongruent) trial.

Importantly, besides intensity, "selectivity" may also constitute an adaptive dimension of control. For instance, while driving a car, events on the sidewalk are usually unimportant, but it might be good to also consider pedestrians on the sidewalk when one is approaching a crosswalk. In addition, in a congruent Stroop trial, it is efficient to use both task-relevant (color) and task-irrelevant (word) dimensions for responding (Melara \& Algom, 2003). Hence, in cases where conflict is low, an agent might consider using both task-relevant and task-irrelevant information. This would result in faster responses when the next trial is congruent but slower responses when the next trial is incongruent.

Furthermore, the time scale at which the conflict signal is applied has been scrutinized (Braver, 2012; Scherbaum, 
Dshemuchadse, Ruge, \& Goschke, 2012; Scherbaum, Fischer, Dshemuchadse, \& Goschke, 2011). In most accounts, control is adapted across trials (slow time scale). In these accounts (Botvinick et al., 2001), if conflict on the current trial was high, the model would exert more intensive control on the next trial. Typically, slow control adaptation is considered in the literature as an instance of proactive control; it is used to prepare for situations that might require control in the (near) future, such as when approaching a crosswalk by car, or the potential incongruency on the next trial of a Stroop task. However, in some situations, proactive control might not suffice and a faster, more urgent control adaptation might be required (fast time scale). For example, a pedestrian may unexpectedly decide to cross the road, or an incongruent Stroop trial may be presented when the participant instead anticipated a congruent one. Such fast control adaptation is defined as an instance of reactive control.

Unfortunately, how cognitive control is mechanistically implemented at a neurophysiological level remains ambiguous. Relatedly, current models of cognitive control typically do not formulate hypotheses about the underlying neurophysiology. One prominent idea, however, considers the role of neural oscillations and, in particular, neural synchrony. More specifically, the binding-bysynchrony (BBS) theory (Fries, 2005, 2015) argues that neural groups with synchronized oscillations can communicate efficiently, whereas neural groups that are desynchronized cannot, or at least less efficiently. Hence, processing in synchronized pathways is prioritized over that in other pathways. In addition, it has been suggested that oscillations in medial frontal cortex (MFC) at theta frequency $(\sim 4-8 \mathrm{~Hz})$ function as a conductor, orchestrating synchrony in more posterior task-relevant networks where activity oscillates at faster (gamma [>40 Hz]) frequencies (for reviews, see Helfrich \& Knight, 2016; Cavanagh \& Frank, 2014). These hypotheses are supported by a broad range of research describing prefrontal theta activity and/or theta-gamma cross-frequency coupling during tasks requiring cognitive control (van Driel, Swart, Egner, Ridderinkhof, \& Cohen, 2015; Voloh, Valiante, Everling, \& Womelsdorf, 2015; Voytek et al., 2015; Nigbur, Cohen, Ridderinkhof, \& Stürmer, 2011; Cavanagh, Frank, Klein, \& Allen, 2010; Hanslmayr et al., 2008; Engel, Fries, \& Singer, 2001). Critically, also midfrontal theta power shows a conflict-adaptation effect (Pastötter, Dreisbach, \& Bäuml, 2013). Specifically, theta power was influenced by the congruency of trial $n$ as well as the congruency of trial $n-1$. In summary, the human brain might implement cognitive control via prefrontal, top-down theta signals that prioritize task-relevant processing by synchronizing gamma activity in posterior processing areas.

Besides behavioral measures, these electrophysiological signatures provide useful empirical constraints to inform our understanding of several aspects of cognitive control, such as its intensity, selectivity, and time scale. To achieve this goal, we need the right conceptual tools, however, and so we implement a conflict monitoring loop (Botvinick et al., 2001) in a recently proposed computational model of neural synchrony (Verguts, 2017). In line with previous suggestions, the measure of conflict is evaluated on slow and fast time scales (Braver, 2012) and is used to adapt the intensity (Botvinick et al., 2001) and/or selectivity (Melara \& Algom, 2003) of control. More specifically, in the current model (further referred to as sync model), a control unit uses top-down biasing signals at theta frequency to synchronize task-relevant gamma activity in the model processing unit. Hence, it implements control via BBS. This allows the model to make empirical contact with neurophysiological data. The control unit of the sync model consists of two parts. Here, the MFC sends bursts at theta frequency. The intensity of control is determined by the number of bursts that are sent by the MFC. Eligible task-specific neural areas that oscillate at gamma frequency become synchronized by the bursts. Which areas are eligible to receive the bursts is determined by the lateral frontal cortex (LFC), which holds task demands (Miller \& Cohen, 2001) and sends an eligibility trace to task-relevant areas. In summary, the MFC determines the intensity of control, and the LFC determines the selectivity of control.

To summarize, the current work aims to evaluate relevant dimensions and time scales for adaptive cognitive control. For this purpose, a novel sync model is proposed in which a conflict monitoring unit evaluates response conflict. This measure of conflict is then used by the control unit of the sync model to adapt the intensity (in the model MFC) and/or selectivity (in the model LFC) of control. In addition, we implement two time scales for adaptive control. On the slow time scale, control is adapted between trials and used proactively to prepare for the next trial (proactive control). On the fast time scale, control is employed in a reactive way to quickly respond to conflict upon stimulus appearance (reactive control). The resulting model allows us to study the role of intensity, selectivity, and time scale in cognitive control, at both behavioral and neurophysiological levels.

\section{METHODS}

\section{The Model}

\section{Overview}

A general overview of the model is given in Figure 1. This model consists of four units. One of these units is a conflict monitoring unit that we adapted from Botvinick et al. (2001). Here, we implemented this conflict monitoring unit in a previously proposed model (Verguts, 2017), which contains a processing unit, a control unit, and an integrator unit. The processing unit contains stimulus and response modules. Each module holds nodes related to one stimulus or response feature (e.g., color or word in a Stroop task). The integrator unit has one node corresponding to each node in the response module. These 
Figure 1. Model architecture. The model consists of four units (dashed boxes). The processing unit holds three task modules. Each task module contains four color nodes that consist of neuronal triplets (square nodes) such as presented in B. In addition, the MFC node in the control module holds a neuronal triplet. Other model nodes (circles and ellipses) only contain rate code neurons. The integrator unit integrates evidence for each response option over time until it reaches a threshold and gives a response. Here, weights

implement lateral inhibition. The conflict monitoring unit evaluates conflict in the integrator unit. The control unit uses this evaluation to adapt when and where to synchronize in the processing unit. Here, the MFC sends synchronizing bursts (dashed line), and the LFC sends eligibility traces (dotted lines) that indicate which modules should be synchronized.

integrator unit nodes integrate evidence over time for the corresponding node in the response module. When this evidence reaches a "threshold" value, the model will give a response. In addition, the conflict monitoring unit measures conflict in the integrator unit. This measure of conflict is then transformed into two values of control. The proactive control node evaluates conflict on a slow (trial-by-trial) time scale. The reactive control node evaluates conflict on a faster (within-trial) time scale, to which the measure of proactive control is added (see Figure 1). Thus, proactive control determines a baseline level of control before every trial. During the trial, if this control was not sufficient to eliminate conflict, the conflict signal will further increase reactive control.

On every time step within a trial, the reactive control signal is transferred to the control unit. This control unit consists of an MFC node and an LFC node, which jointly implement control by sending synchronizing bursts to the modules in the processing unit. Here, the MFC determines the intensity of the bursts. The stronger the control signal it receives from the conflict monitoring unit, the more bursts it will send. The LFC determines the selectivity of the bursts. When the control signal is strong, the LFC node will indicate that only the relevant stimulus module and response module should receive bursts (selective control). If the control signal is weaker, indicating that there was little conflict, the LFC will make all modules eligible to receive bursts (nonselective control). In this way, we implement earlier suggestions that, in cases of low conflict, task-irrelevant information might be processed as well (Melara \& Algom, 2003). Below, and in Figure 2, we elaborate on the computational implementation of the model.

\section{Processing Unit}

In the context of a Stroop task, the processing unit consists of three modules; two stimulus modules (color and word) and one response module. Each module contains four nodes (one for each color in the Stroop task) that each hold one neuronal triplet. In the sync model, each neuronal triplet contains one excitatory-inhibitory pair of phase code neurons $(E, I)$, which appears in combination with a rate code neuron ( $x$; see Verguts, 2017, for details). In line with previous work (Verguts, 2017), excitatory neurons are updated by

$$
\Delta E_{i}(t)=C I_{i}(t)-D \times J\left(r>r_{\text {min }}\right) \times E_{i}(t)+B_{i}(t)
$$

where $\Delta E(t)=E(t+\Delta t)-E(t)$, and inhibitory neurons are updated by

$$
\Delta I_{i}(t)=-C E_{i}(t)-D \times J\left(r>r_{\text {min }}\right) \times I_{i}(t)
$$

Hence, activation in both neurons is updated on every time step $(\Delta t=2 \mathrm{msec})$. This update crucially relies on the coupling parameter $C$. More specifically, phase code neurons will oscillate at a frequency of $C / 2 \pi$. Here, $C$ was chosen such that oscillations in the two stimulus modules were at a frequency of $40 \mathrm{~Hz}$, whereas oscillations in the response module were at $39 \mathrm{~Hz}$. Thus, there was a small frequency difference of $2.5 \%$ between stimulus and response modules; as a result, oscillations would drift apart (desynchronize) in the absence of control. The terms $D \times$ $J\left(r>r_{\text {min }}\right) \times E(t)$ in Equation 1 and $D \times J\left(r>r_{\text {min }}\right) \times I(\mathrm{t})$ in Equation 2 attract the radius of oscillations toward a value of $r_{\min }=1$ (see also Verbeke \& Verguts, 2019; Verguts, 2017). In the Appendix, we present results for different frequencies in both the control and processing units.

Excitatory $(E)$ neurons in the processing unit additionally receive a burst B (see also Figure 2). These bursts are described by

$$
B_{i}(t)=L F C_{i}(t) \times M F C_{i}(t) \times U(t)
$$

As described in previous work (Springer \& Paulsson, 2006; Verguts, 2017; Zhou, Chen, \& Aihara, 2005), 
oscillators that receive positively correlated noise (or input signals, more generally) will become synchronized. Hence, the sync model will synchronize modules (and hence nodes) that receive the same LFC and MFC signal. Here, $U$ represents standardized Gaussian noise.

The rate code neurons in the processing unit are updated by

$$
\Delta x_{i}(t)=-x_{i}(t)+\left(Z_{i}+\mathbf{W}_{i}^{T} \boldsymbol{x}(\boldsymbol{t})+S(t)\right) \times G\left(E_{i}(t)\right)
$$

The term $-x_{i}(t)$ will cause fast decay of activation in the absence of input. The variable $Z_{i}$ represents external input that is received by rate code neuron $i$. On every trial, two input nodes, one in each stimulus module, receive an input of 1 , whereas all other nodes receive external input 0 .
The term $\boldsymbol{W}_{\boldsymbol{i}}^{T} \boldsymbol{x}(\boldsymbol{t})$ represents internal input to $x_{i}$. Here, $\mathbf{W}_{\boldsymbol{i}}$ is a (column) vector representing the weight (strength) between $x_{i}$ and all other rate code neurons (represented in vector $\boldsymbol{x}$ ). Each node in the stimulus modules projects to one node in the response module (weight different from zero) that represents the same color. Hence, every color node in the response module receives (nonzero) input from two input nodes (one from the color module and one from the word module). To implement the empirically observed imbalance in the tendency to respond to the word rather than the color dimension (Cohen et al., 1990), weights $(\boldsymbol{W})$ between the word and response modules have a strength of 1 , whereas weights between the color and response modules have a smaller strength of .9. As a manipulation check, we performed simulations of the

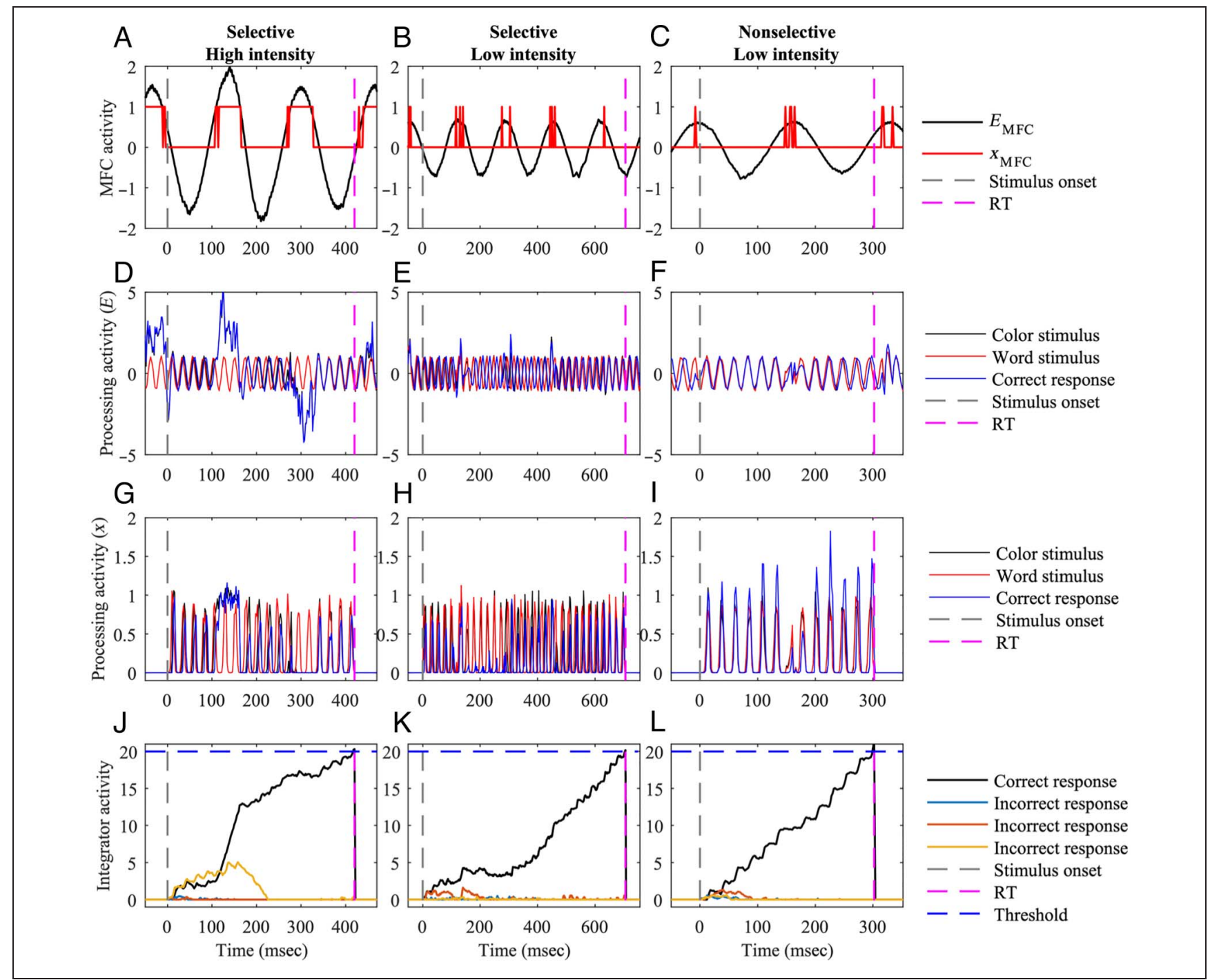

Figure 2. Model implementation. We show activity in the MFC, the excitatory neurons of the processing unit, the rate code neurons of the processing unit, and the integrator unit (rows) for three types of trials (columns) from 50 msec before stimulus onset until 50 msec after RT. Notice that this means that the $x$ limits are different for each column. In the first column (A, D, G, and J), activity is shown for a trial in which selective control is applied with high intensity. Here (A), $E_{\mathrm{MFC}}$ has a high amplitude that leads to more bursts $\left(x_{\mathrm{MFC}}=1\right)$. This results in synchrony and more efficient communication between the color and correct response neurons. Therefore, the correct response wins the competition in the integrator unit. When the intensity of control is lower (second column; B, E, H, and K), less bursts are provided (E), which leads to less efficient communication $(\mathrm{H})$ and slower RTs $(\mathrm{K})$. When control is nonselective (C, F, I, and L), all nodes in the processing unit (word, color, and response) get synchronized (F), which leads to faster RTs in congruent trials (L). Note that for control to be nonselective, the intensity should always be low. 
nine model versions (see Simulations). As we expected, this unbalanced weight matrix led to faster RTs when the models were required to respond to the word ( $M=$ $382.5 \mathrm{msec})$ than to the color $(M=418 \mathrm{msec})$. In what follows, we only report simulations in which the model was required to respond to color. In addition, on each time step, some Gaussian noise $(S(t))$ is fed to the rate code neuron with a mean of 0 and a standard deviation of 0.1 . The sum of noise, external, and internal input is multiplied in Equation 4 by a function of the excitatory phase code neuron:

$$
G\left(E_{i}(t)\right)=\frac{1}{1+e^{\left(-5 \times\left(E_{i}(t)-.6\right)\right)}}
$$

Because of this gating function $\left(G\left(\mathrm{E}_{i}(t)\right)\right)$, activation of the rate code neurons is strongest when the excitatory neuron is near its top. When the excitatory neuron is at its trough, $\mathrm{G}(E(t))$ is close to zero and activation in the rate code neurons is suppressed.

\section{Integrator Unit}

In addition, the integrator unit contains four color nodes. For simplicity, nodes in the integrator unit contain only rate code neurons. These neurons integrate evidence from the output layer of the processing unit over time by implementing a competitive accumulator model (Usher \& McClelland, 2001) via

$$
\Delta y_{i}(t)=\boldsymbol{A}_{i}^{T} \boldsymbol{x}(\boldsymbol{t})+\boldsymbol{L}_{i}^{T} \boldsymbol{y}(\boldsymbol{t})+S(t)
$$

Here, $\boldsymbol{A}_{\boldsymbol{i}}$ is a column vector, representing connections between nodes in the processing unit and node $i$ of the integrator unit. Each node in the response module of the processing unit is connected to one node in the integrator unit with a strength of .5; all other weights are set to zero. $\boldsymbol{L}_{i}$ corresponds to column $i$ of Matrix $\boldsymbol{L}$, which has a diagonal of zero with off-diagonal elements, -.015 . This implements lateral inhibition between integrator nodes $(\boldsymbol{y}) . S(t)$ again implements Gaussian noise with a mean of 0 and a standard deviation of 0.1 . When one of the integrator nodes reaches a threshold of 20, the model will respond. An illustration of the integrator dynamics is provided in Figure 2.

\section{Conflict Monitoring Unit}

The conflict monitoring unit evaluates conflict in the integrator unit. In line with previous work (Botvinick et al., 2001), conflict is defined as energy of activity in the integrator unit.

$$
\text { Conflict }(t)=\frac{1}{\text { threshold }} \times \sum_{i=1}^{I-1} \sum_{j=i+1}^{I} y_{i}(t) \times y_{j}(t)
$$

Here, multiplied activation in each integrator pair is added. Hence, if only one integrator neuron is active,
Conflict $=0$. For standardization, activation is divided by the threshold parameter.

In addition, in line with previous work (Botvinick et al., 2001), Conflict is employed to adjust control by the following rule

$$
\begin{aligned}
\Delta_{\text {Control }_{t s}(T)=} & \gamma_{t s} \times\left(\beta \times \varepsilon_{t s}+\eta_{t s}\right)-\left(1-\gamma_{t s}\right) \\
& \times \operatorname{Control}_{t s}(T)
\end{aligned}
$$

As mentioned before, Control is adapted on two time scales ( $t$ s $\in$ \{reactive, proactive $\}$ ), by computing a weighted integration of the time-scale-specific conflict signal $\varepsilon_{t s}$. First, for proactive control (slow time scale; $t=$ trial $(n)$ ), max (Conflict $(n))$ is used as the conflict signal $\varepsilon_{\text {proactive }}$ to determine a baseline control intensity on the next trial $(n+1)$. Here, $\eta_{\text {proactive }}=.25$. Second, reactive control is adjusted on a fast time scale $(T=$ time steps $(t))$, where $\varepsilon_{\text {reactive }}=$ Conflict $(t)$. Crucially, reactive control uses proactive control as a baseline, in particular, $\eta_{\text {reactive }}=$ Control $_{\text {proactive }}$ (n). The parameter $\beta$ is the same for both time scales and is set to 10 . Values for $\gamma_{\text {proactive }}$ and $\gamma_{\text {reactive }}$ differed across model versions (see below). Note that, when $\gamma_{t s}=$ 0 , control does not change on that time scale.

\section{Control Unit}

The control unit consists of an LFC node and an MFC node. Both nodes use the conflict signal on the fast time scale to adjust how they will exert top-down modulation to pathways in the processing unit. As in the processing unit, the MFC contains a neuronal triplet. Here, phase code neurons oscillate at a $6-\mathrm{Hz}$ (theta) frequency and $B_{\mathrm{MFC}}(t)=0$. In addition, the power $\left(r_{\min }\right)$ of the oscillation is adjusted on every time step by

$$
r_{\min }(t)=1+\theta_{\text {intense }} \times\left(\text { Control }_{\text {reactive }}(t)-1\right)
$$

The (binary) parameter $\theta_{\text {intense }}$ determines whether power intensity changes across time; if $\theta_{\text {intense }}=0$, then $r_{\min }$ (and hence MFC power) remains fixed at 1 ; otherwise, $r_{\min }$ (and hence MFC power) increases when Control $_{\text {reactive }}$ increases.

The MFC rate code neuron obeys a Bernoulli process, which is 1 with probability $p(t)$. The probability

$$
p(t)=\frac{1}{1+e^{\left(-10 \times\left(E_{\mathrm{MFC}}(t)-.8\right)\right)}}
$$

is a sigmoid function that attains its highest value when $E_{\mathrm{MFC}}$ is at its top. Hence, every time the oscillation of the $E_{\mathrm{MFC}}$ neuron reaches its top, the probability of a burst becomes high. Moreover, this top itself increases with increasing power; thus, when power in the MFC is high, the probability for bursts remains high for a longer period. Hence, with higher power, the MFC will send more bursts to the processing unit and accomplish better synchronization. In summary, as in previous work (Botvinick et al., 2001), the intensity of control is increased when more conflict is detected. We provide examples of simulated trials in Figure 2. Figure 2A illustrates 
MFC activity during a high-conflict (see Equation 7) trial. As can be observed, MFC theta power increases after stimulus onset. When power is high (Figure 2A), more bursts $\left(x_{\mathrm{MFC}}=1\right)$ are sent than when power is low (Figure 2B and C). Bursts are observed in the excitatory neurons of the processing unit (Figure 2D) where the color stimulus (but not the word stimulus) and correct response become synchronized. This leads to efficient communication between the color and response rate code neurons (Figure 2G). Hence, despite competition from incorrect response nodes, the integrator node for the correct response reaches the decision threshold first (Figure 2J). When the intensity of control is lower (Figure 2B), less bursts are provided (Figure 2E), which leads to less efficient communication (Figure $2 \mathrm{H}$ ) and slower RT (Figure 2K). Important to note is that we refer to the bursts as noise, in the sense that the input signal (from MFC) should not contain any information; receiving the signal does not lead to noisier (less efficient) communication between nodes that receive the bursts. In fact, we observe in Figure 2J a faster evidence accumulation during the bursts (Figure $2 \mathrm{G}$ ) than before or after the bursts.

In the context of a Stroop task, the model holds three LFC nodes (only rate code neurons) that each project to one task module in the processing unit. The model always had to respond to the font color. Therefore, activation of $L F C_{\text {Color }}$ and $L F C_{\text {Response }}$ is fixed to 1 . Hence, the color and response modules are always selected to receive bursts and therefore always become synchronized. In contrast,

$$
\operatorname{LFC}_{\text {word }}(t)=J\left(\text { Control }_{\text {reactive }}(t)<\theta_{\text {selective }}\right)
$$

in which $J($.$) is an indicator function returning 1$ when Control $_{\text {reactive }}(t)<\theta_{\text {selective }}$ and 0 otherwise. Thus, when the conflict monitoring unit judges that not a lot of control is necessary, all three modules in the processing unit are synchronized. This corresponds to applying control to all task modules (nonselective control). In these trials, the model will also use information from the taskirrelevant (word) dimensions to reach a faster response. When Control reactive is high, $L F C_{\text {Word }}=0$ and control is only applied to the response and relevant stimulus module (selective control). In these trials, the model aims to avoid interference from the color dimension and only considers information from the word dimension. Hence, when more conflict is experienced, control will be applied more selectively. Whereas higher values of $\theta_{\text {selective }}$ will result in a higher probability of nonselective control, lower values will reduce the probability of nonselective control. When $\theta_{\text {selective }}<\eta_{\text {proactive, }} L F C_{\text {Word }}$ can never be 1 . As a result, only selective control will be employed. Again, we provide in Figure 2 examples of simulated trials in which either selective or nonselective control was applied. This figure illustrates how for selective control only the task-relevant (color) neuron is synchronized with the response node (Figure 2E), whereas for nonselective control, all oscillations in the processing unit are synchronized (Figure 2F). For congruent trials, nonselective control will lead to faster RT (Figure 2I).

\section{Simulations}

Nine versions of the model were simulated. Here, we evaluated the intensity and selectivity dimensions as well as the proactive and reactive time scales of adaptive cognitive control. More specifically, adaptive control was explored for three possibilities of adaptive dimensions (intensity, selectivity, or both) and three possibilities of time scale (fast, slow, or both), resulting in nine possible combinations. Parameter values that defined each model version are presented in Table 1 . We performed extra simulations for every model version with different parameter settings. Although conflict-adaptation effects might quantitatively differ across these parameter values (see also the Appendix), effects that are presented in the Results section as not significant were nonsignificant across all parameter settings for that model version, thus attesting to the robustness of our simulations.

For each model version, 30 simulations were performed of 600 trials (300 congruent) each. Each trial consisted of an intertrial interval (ITI) of $500 \mathrm{msec}$ in which no external input was provided to the model and a trial period of $2000 \mathrm{msec}$ in which external input was presented until the model gave a response. After this response, all activation in the integrator unit was reset to 0 .

Table 1. Parameter Settings for the Nine Different Model Versions (Columns)

\begin{tabular}{|c|c|c|c|c|c|c|c|c|c|}
\hline & Proactive & Proactive & Proactive & Reactive & Reactive & Reactive & Both & Both & Both \\
\hline & Intensity & Selectivity & Both & Intensity & Selectivity & Both & Intensity & Selectivity & Both \\
\hline$\gamma_{\text {proactive }}$ & .25 & .25 & .25 & 0 & 0 & 0 & .75 & .75 & .75 \\
\hline$\gamma_{\text {reactive }}$ & 0 & 0 & 0 & 1 & 1 & 1 & 1 & 1 & 1 \\
\hline$\theta_{\text {intense }}$ & 1 & 0 & 1 & 1 & 0 & 1 & 1 & 0 & 1 \\
\hline$\theta_{\text {selective }}$ & 0 & .75 & .75 & 0 & .75 & .75 & 0 & .75 & .75 \\
\hline
\end{tabular}

Column headings indicate time scale (proactive, reactive, or both) and control dimension (intensity, selectivity, or both). 


\section{Analyses}

For all trials across all simulations, the model reached a response threshold within the 2000-msec deadline. However, before analyses, the first trial of every simulation was removed because we could not evaluate this trial with respect to the congruency on trial $n-1$. In addition, to deal with the skewed RT distributions, the natural $\log$ of RT was used in all analyses.

\section{Behavioral}

For behavioral analyses, we employed linear mixed models to analyze RT data and generalized linear mixed models to analyze accuracy. For each model version, a random intercept across simulations was fitted together with fixed slopes for the congruency on trial $n$ and congruency on trial $n-1$ as well as the interaction between congruency on trial $n$ and trial $n-1$.

To link the simulated data of our model to previous empirical findings, we reanalyzed data from Experiment 1 in Schmidt and Weissman (2014). This article provided an open access data set that allowed us to compute the mean behavioral performance (accuracy and RT) for all four combinations of congruency on trial $n$ and trial $n-1$. Similar as for the simulations, we removed the first trial of every experimental run and trials for which the RT was slower than $2000 \mathrm{msec}$. In addition, we used the natural log of RT for the analyses.

\section{Power}

Although in typical EEG studies, power is analyzed by baselining it with respect to activation in the ITI, the model does not have to deal with noise in the data and therefore also allows us to analyze raw (unbaselined) power. We distinguish between three measures of power in the MFC. First, we analyze power during the ITI. In this period, there is no conflict (measured via response energy). Hence, power is only determined by proactive control. Second, there is power during the trial itself. This corresponds to the reactive control signal. Third, we investigate baselined power. Here, we adopt the classical approach of EEG studies by baselining trial power with respect to the ITI. Note that, although power is most often baselined with respect to the power within a pretrial period averaged across all trials, the current work adopted the approach of Pastötter et al. (2013) to baseline power with respect to pretrial power of only one trial. This allows us to investigate baselined reactive control, isolated from the proactive control signal that is present in unbaselined reactive control. We analyze baselined power to connect to empirical data but use unbaselined power to provide additional insights into the model dynamics.

Power was decibel transformed by

$$
\text { Power }=10 \times \log _{10}\left(P_{\text {period }}\right)
$$

in which for the trial and ITI period,

$$
P_{\text {period }}=\sum_{t=s}^{R}\left|E_{\mathrm{MFC}}(t)\right|^{2} /(R-s)
$$

with $s=1$ and $R=500$ (500 msec; end of ITI) in the ITI period and with $s=501$ and $R=$ RT in the trial period. For the baselined power, $P_{\text {baselined }}=P_{\text {trial }} / P_{\text {ITI }}$.

To connect to empirical data, we also ran linear mixed model regressions with baselined power as the dependent variable and with congruency on trial $n$ and congruency on trial $n-1$ as the independent variables. Again, a random intercept was fitted across simulations.

\section{Neural Synchrony}

The current model allows us to test the selectivity of control by measuring neural synchrony between the stimulus and response modules in the processing unit. However, in contrast to power, the critical neural synchrony tests for our model have not been reported yet in the literature. Indeed, in contrast to previous work (Hanslmayr et al., 2008), we analyzed neural synchrony between task-related posterior processing areas. We do not consider synchrony between frontal control areas, because only one of our frontal areas exhibited oscillations. In the model, selective control would entail that only the color module is synchronized with the response module, whereas nonselective control would result in synchrony between both stimulus modules and the response module. Synchrony between two nodes is measured as the correlation over time of activation in both excitatory neurons. This results in a value of synchrony for each input-output pair on each trial. To increase testability of the model predictions with EEG, which typically has a low spatial resolution, we averaged synchrony over all stimulus-response pairs. This results in one value of synchrony between stimulus modules (e.g., posterior visual regions) and the response module (e.g., lateral motor regions) on every trial. When nonselective control is applied, the average synchrony should be higher than when selective control is applied.

Again, a linear mixed model was tested with neural synchrony as the dependent variable, congruency on trial $n$ and congruency on trial $n-1$ as the independent variables, and a random intercept across simulations.

\section{Relations between Neural and Behavioral Data}

To link behavioral data to the underlying neural mechanisms, we again employed linear mixed models. Specifically, we tested three mixed models for both RT and accuracy (six models altogether). Because accuracy data are binary, generalized linear mixed models were used in this case. In each model, a random intercept across simulations was included together with one of three neural measures (proactive and reactive power and one of synchrony). In addition, the interaction between the neural measure and congruency on trial $n$ was tested. 


\section{RESULTS}

\section{Reproducing Empirical Conflict-Adaptation Effects}

As described before, the main interest of the current study is the conflict-adaptation effect. This effect is typically represented as an interaction between congruency on trial $n$ and congruency on trial $n-1$ (but see also Braem et al., 2019). More specifically, previous work has observed that performance improves (higher accuracy and faster RT) when the congruency of trial $n$ matches the congruency of trial $n-1$ (see Figures 4J and 5G; e.g., Schmidt \& Weissman, 2014; Gratton et al., 1992). In addition, at the neurophysiological level, previous work illustrated that midfrontal theta power is lower when the congruency of trial $n$ matches the congruency of trial $n-1$ (Figure 6E; Pastötter et al., 2013). We evaluated nine model versions that explored different dimensions and time scales of control adaptation. Conflict-adaptation effects for all nine model versions (see Table 1) and all three data modalities (accuracy, RT, and power) were expressed as $\chi^{2}$ statistics for the interaction of congruency on trial $n$ and trial $n-1$. Raw statistics ranged from 0.05 to 1776 for accuracy, from 0.014 to 3477 for RT, and from 0.009 to 1958 for power. To compare the model versions, statistics were $z$ scored across model versions, for each data modality separately. The resulting normalized fit measures (i.e., evidence for a conflict-adaptation effect) are summarized in Figure 3 (see also van den Berg, Awh, \& Ma, 2012, for a similar approach).

Below, we explore the model results in more detail. We start by evaluating conflict-adaptation effects in accuracy for all nine model versions. To avoid redundancy of presenting results from unsuited model versions, we consistently eliminate model versions that could not reproduce conflict-adaptation effects in one data modality before moving on to the next modality. We aim to define the minimal requirements for control adaptation to reproduce empirical conflict-adaptation effects in all three modalities.

\section{Accuracy}

Accuracy results for all nine versions of all model versions are shown in Figure 4. All nine models showed a significant effect of congruency on trial $n$ [all $p s<.001$, all $\chi^{2}(1, n=$ $30)>158]$. Six of the nine model versions exhibited significant effects of congruency on trial $n-1$ [all $p$ s $<$ .001 , all $\chi^{2}(1, n=30)>18$ ] and significant interactions between congruency on trial $n$ and congruency on trial $n-1$ [all $p$ s $<.001$, all $\chi^{2}(1, n=30)>18$ ]. As illustrated in Figure 4, models that only employed reactive control (Figure 4D-F; fast time scale) could not account for conflict-adaptation effects from empirical studies (Figure 4J). Hence, these models were disregarded for further evaluations (but see Figure 3). In summary, a minimal requirement for reproducing conflict-adaptation effects in accuracy is employing proactive control (slow time scale).

\section{$R T$}

The results of all nine model versions are summarized in Figure 3. For illustrative purposes, we only evaluated results of RT analyses for the six models that illustrated a significant conflict-adaptation effect in accuracy (see Figure 5). Also here, all models showed a significant effect of congruency on trial $n$ [all $p$ s $<.001$, all $\chi^{2}(1, n=30)>13,974$ ]. Moreover, all remaining models had a main effect of congruency on trial $n-1$ [all $p$ s $<.0025$, all $\chi^{2}(1, n=30)>$ 9.19]. Importantly, although also the interaction between congruency on trial $n$ and trial $n-1$ reached statistical significance in these six models [all $p s<.002$, all $\chi^{2}(1$, $n=30$ ) $>9.71$, only four versions (Figure 5B, C, E, and $\mathrm{F}$ ) of the model reproduced the qualitative pattern that is typically found in empirical data (Figure 5G), where there are performance benefits on congruent-congruent trial sequences. In summary, according to model simulations, minimal requirements for producing a conflict-adaptation effect in $\mathrm{RT}$ are proactive control (slow time scale) and adaptivity at the selectivity dimension. When this is combined with reactive control or with adaptivity at the intensity dimension, the conflict-adaptation effect remains intact. Although the intensity dimension (Figure 5A and D) was sufficient to explain performance improvements for incongruentincongruent trial sequences, these models were not able

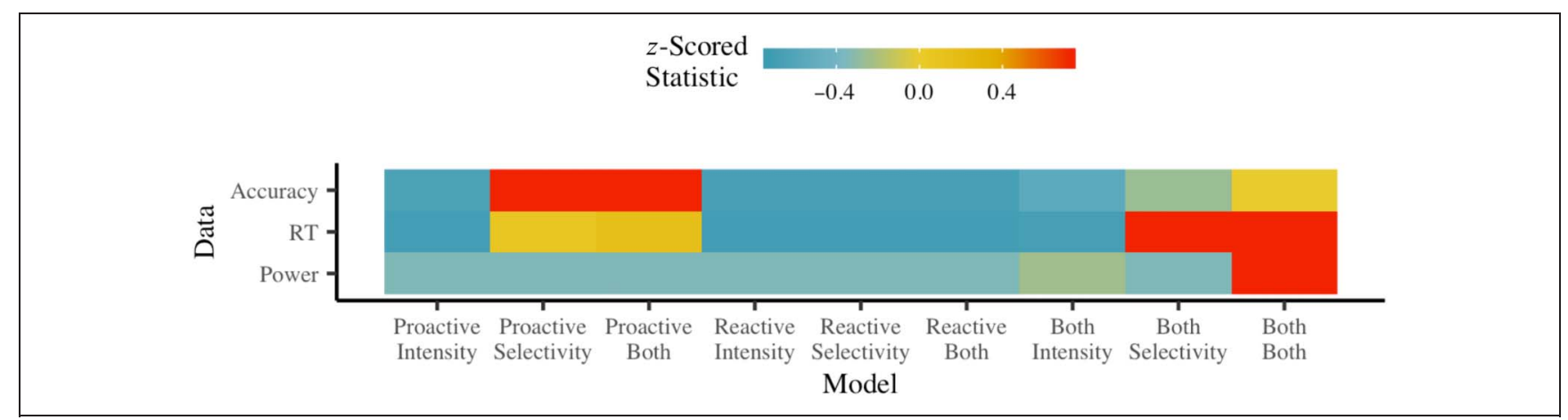

Figure 3. Conflict-adaptation effects for each data modality and each model. Columns represent model versions (same order as in Table 1), and rows present the three data types that were used to compare models. Colors represent the statistic $\left(\chi^{2}\right)$ values for the interaction effect between congruency on trial $n$ and congruency on trial $n-1$. These values were $z$ scored with respect to the other models. 


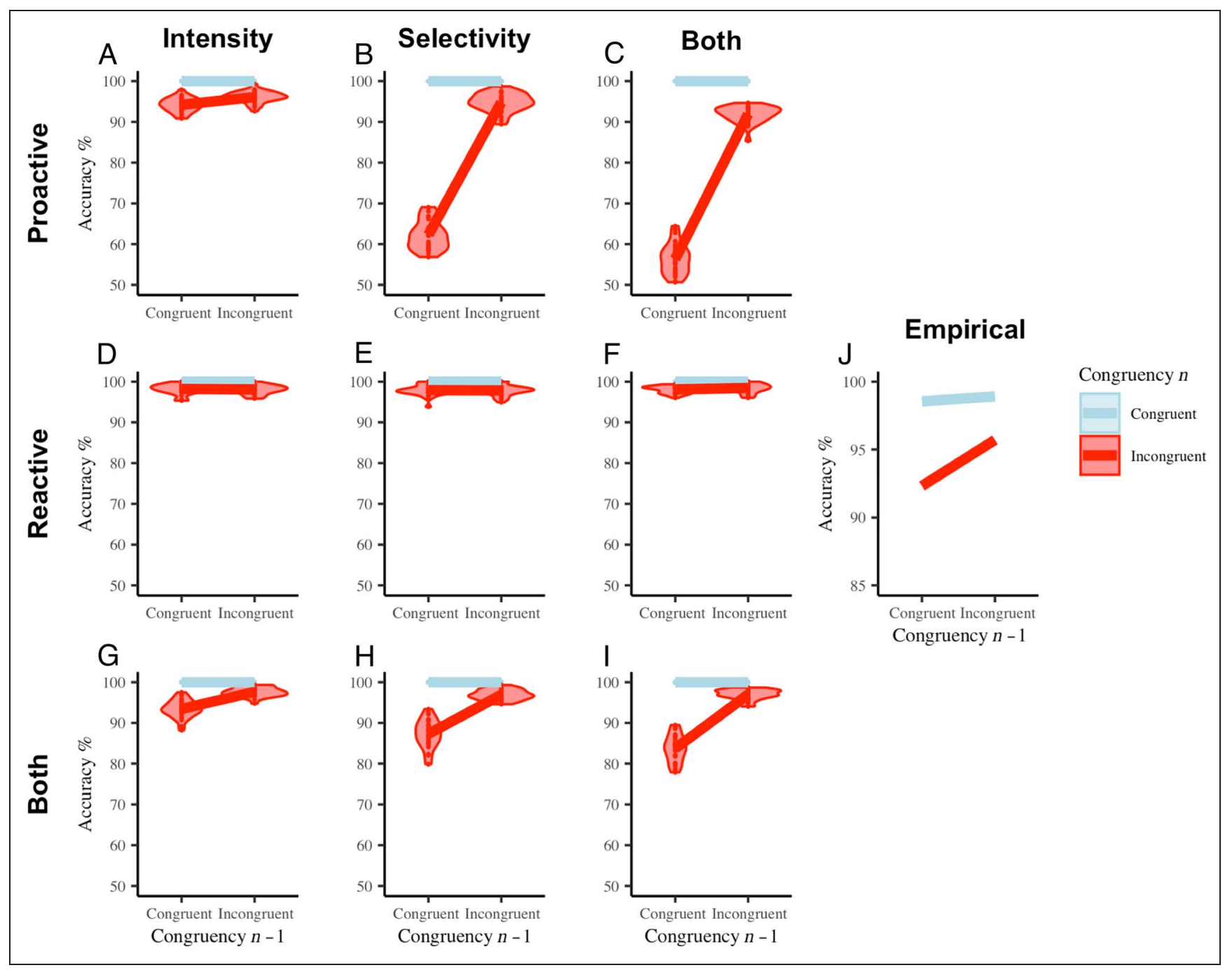

Figure 4. Accuracy results. Lines connect the means for all trials and all simulations; dots represent the mean of one simulation. For A-I, the three rows represent the time scales of adaptive control; the three columns represent the three adaptive dimensions of control. In J, reanalyzed empirical data from Schmidt and Weissman (2014) are shown.

to reproduce the performance benefit of having a congruent-congruent trial sequence that is observed in empirical research (Figure 5G; see also Figure 3 for a summary that these models do not perform well). Hence, four model versions remained for further evaluation.

\section{Power}

Here, we investigated baselined theta power in the MFC of the model. A summary of results for all nine model versions is presented in Figure 3, but for more detailed analyses, only the four versions of the model that could qualitatively reproduce the empirically observed conflictadaptation effect in both accuracy (Figure 4J) and RT (Figure 5G) were considered in our comparison of power results. As presented in Figure 6, only one of the four remaining model versions was able to reproduce the conflict-adaptation effect in midfrontal theta power (Figure 6E; Pastötter et al., 2013). The model that was adaptive on both the intensity and selectivity dimensions and on both time scales (Figure 6D; full adaptive model) illustrated a significant effect in power for congruency on trial $n\left[p<.001, \chi^{2}(1, n=30)=8072\right]$, congruency on trial $n-1\left[p<.001, \chi^{2}(1, n=30)=814\right]$, and the interaction of the two $\left[p<.001, \chi^{2}(1, n=30)=1958\right]$. Apart from the effect of congruency on trial $n-1$ in the proactive model that was adaptive on both dimensions [Figure 6B; $p<.001, x^{2}(1, n=30)=201$ ], no other effects reached significance. Thus, only the full adaptive model, employing adaptivity on both time scales and on both the intensity and selectivity dimensions, survived qualitative comparison to previous empirical data.

\section{Summary}

To summarize, six models showed conflict adaptation in behavior, illustrated by a significant interaction between congruency on trial $n$ and trial $n-1$. These were the models that used control on a slow (proactive) time scale or on both time scales. Importantly, only four of these 


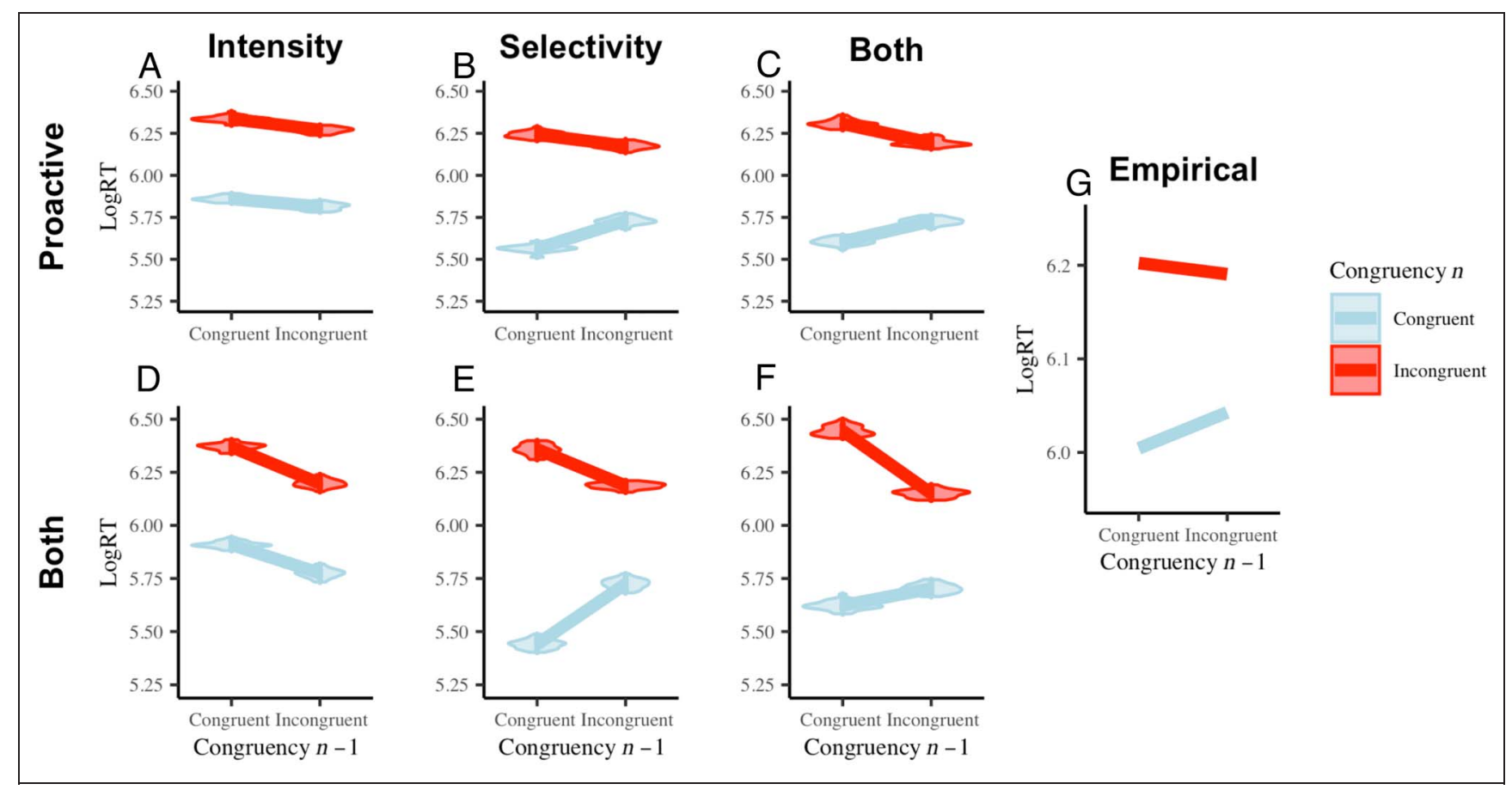

Figure 5. RT results. Lines connect the means for all trials and all simulations; dots represent the mean of one simulation. For A-F, the two rows represent the remaining time scales of adaptive control, and the three columns represent the three adaptive dimensions of control. In G, reanalyzed empirical data from Schmidt and Weissman (2014) are shown.

models could actually reproduce the performance benefits found in congruent-congruent trial sequences (Figure 5G; Egner, 2007). Thus, four models survived comparison to behavioral empirical data. Only one of the four remaining models illustrated a significant conflict adaptation in theta power. Hence, only this model that was adaptive on both time scales and on both intensity and selectivity dimensions could reproduce empirical (behavioral and

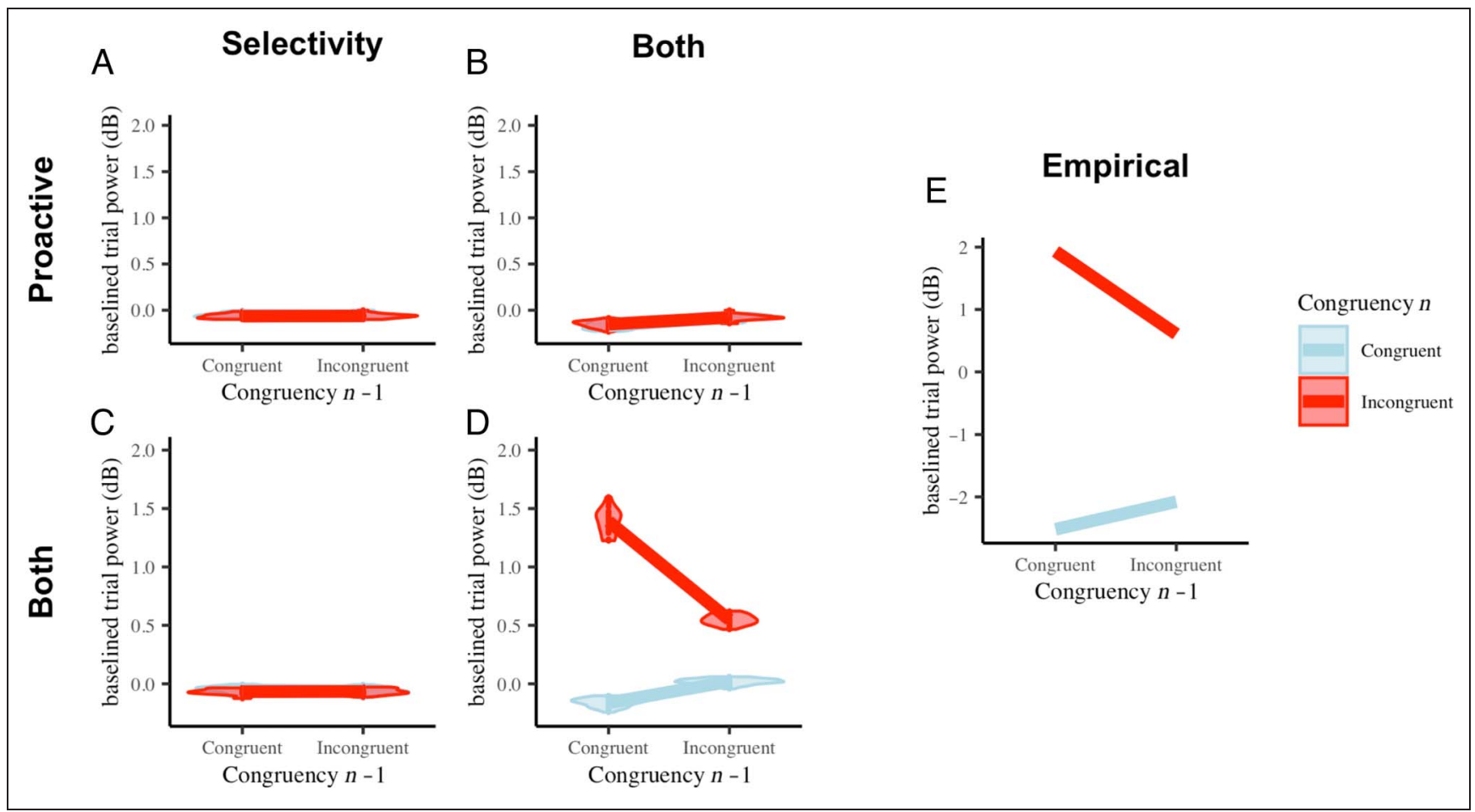

Figure 6. Power results. Lines connect the means of baselined power for all trials and all simulations; dots represent the mean of one simulation. The two rows of A-D represent the remaining time scales of adaptive control; the two columns represent the two remaining adaptive dimensions of control. In E, the reported means from Pastötter et al. (2013) are shown. These values were transformed from percentage change to a decibel scale. 
neurophysiological) conflict-adaptation effects. Therefore, only this full adaptive model is used for consecutive analyses. In the remainder of the Results section, we will increase insight into the model dynamics and provide predictions for future empirical (neurophysiological) work.

\section{Model Dynamics and Predictions}

\section{Power}

To present additional insight in the model dynamics that drove the conflict-adaptation effect in power, Figure 7A and $\mathrm{B}$ shows unbaselined power from the (winning) model MFC during the ITI (Figure 7A) and during the trial period (Figure 7B). Note that power in the MFC represents activation of the Control $_{\text {reactive }}$ node in the conflict monitoring unit (Equation 9). During the ITI, there is no conflict-related activity at response level. As follows from Equation 8, Control reactive $=$ Control $_{\text {proactive }}$ during this ITI period (Figure 7A; proactive power). Hence, MFC power during the ITI represents the proactive control signal that varies on a slow time scale. As a result, Figure 7A only shows an effect of congruency on the previous trial. More specifically, it increases after incongruent trials because the model experienced more conflict during these trials. As can be observed, fast conflict-related activity is added to the

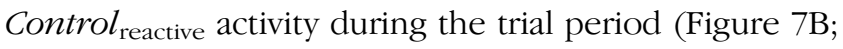
reactive power). Here, activity does not change relative to proactive power (Figure 7A) when the current trial $(n)$ is congruent but does increase when the current trial is incongruent. This fast, conflict-related activity can be isolated by baselining power during the trial period with respect to power in the ITI (see Figure 6E).

\section{Neural Synchrony}

Figure 7C shows results from the neural synchrony analyses. Again, these results provide predictions for synchrony between posterior task-related processing areas, not between frontal control areas (see also Methods and Discussion). All effects reached significance. We found a main effect of congruency on trial $n\left[x^{2}(1\right.$, $n=30)=2246, p<.001$ ], a main effect of congruency on trial $n-1\left[\chi^{2}(1, n=30)=1475, p<.001\right]$, and a significant interaction $\left[\chi^{2}(1, n=30)=1503, p<.001\right]$. As follows from Equation 11, synchrony is strongest when there is least conflict-related activity, which is the case when trial $n-1$ and trial $n$ are both congruent. When conflict exceeds a threshold value ( $\theta_{\text {selective; }}$ see Equation 11), only one stimulus dimension is synchronized and synchrony baselines around 0.5 . Thus, the model predicts an inverse conflict-adaptation effect in neural synchrony between task-related processing areas.

\section{Relations between Neural and Behavioral Data}

To shed further light onto the model dynamics and provide additional predictions for future electrophysiological studies, we performed regressions with congruency (on $\operatorname{trial} N$ ) and neurophysiological data (power or synchrony) as the independent variables and behavioral data as the dependent variable (accuracy or RT). Results are shown in Figure 8.

When we investigate the relation between proactive power and behavior (Figure $8 \mathrm{~B}$ and $\mathrm{F}$ ), all effects reached significance: There was a main effect of proactive power [RT: $\chi^{2}(1, n=30)=1221, p<.001$; accuracy: $\chi^{2}(1, n=$ $30)=555, p<.001]$ and an interaction between proactive power and congruency on trial $n$ [RT: $\chi^{2}(1, n=30)=$ $2805, p<.001$; accuracy: $\left.\chi^{2}(1, n=30)=81.3, p<.001\right]$. Importantly, for incongruent trials, accuracy increased (Figure 8B) and RT decreased (Figure 8F), with stronger power in incongruent trials. In contrast, for congruent trials, RT still increased with higher power values. This was caused by adaptation in the selectivity dimension. When power was low in congruent trials, the model would synchronize all stimulus modules and respond faster (see also Figure $8 \mathrm{H}$ ).

In addition, we analyzed reactive power. Note, however, that reactive power is strongly influenced by proactive power (see Equation 8), as is demonstrated by the fact that reactive power results (Figure $8 \mathrm{C}$ and $\mathrm{G}$ ) mimic those of proactive power analyses (Figure $8 \mathrm{~B}$ and $\mathrm{F}$ ).
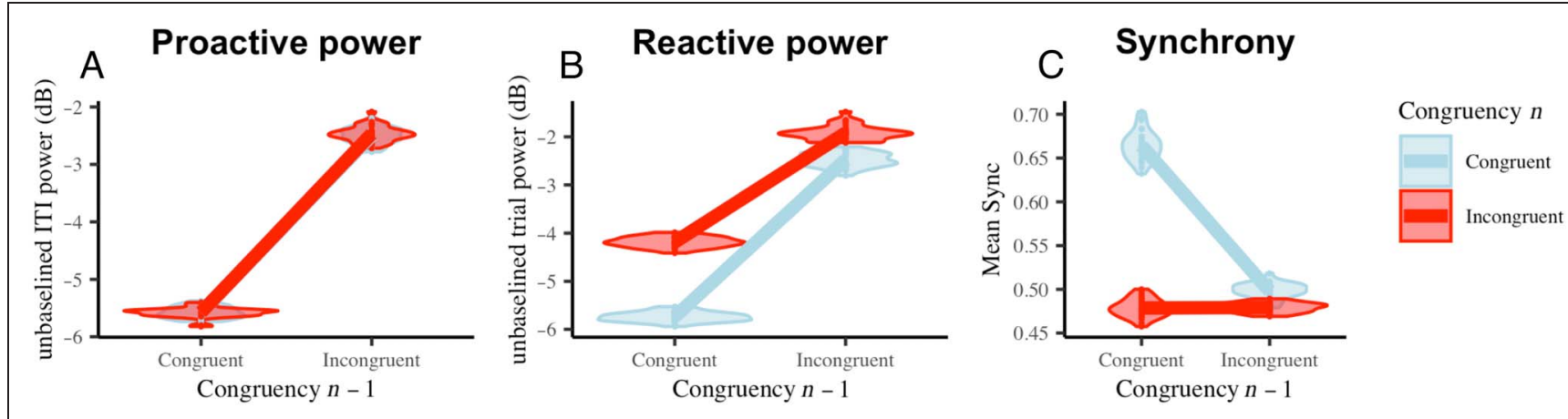

Figure 7. Model dynamics. A illustrates power in the model MFC during the ITI. In B, MFC unbaselined power is presented during the trial (stimulus onset until response). C illustrates neural synchrony between task-related processing areas. Lines represent the mean for all trials and all simulations; dots represent the mean of one simulation. 


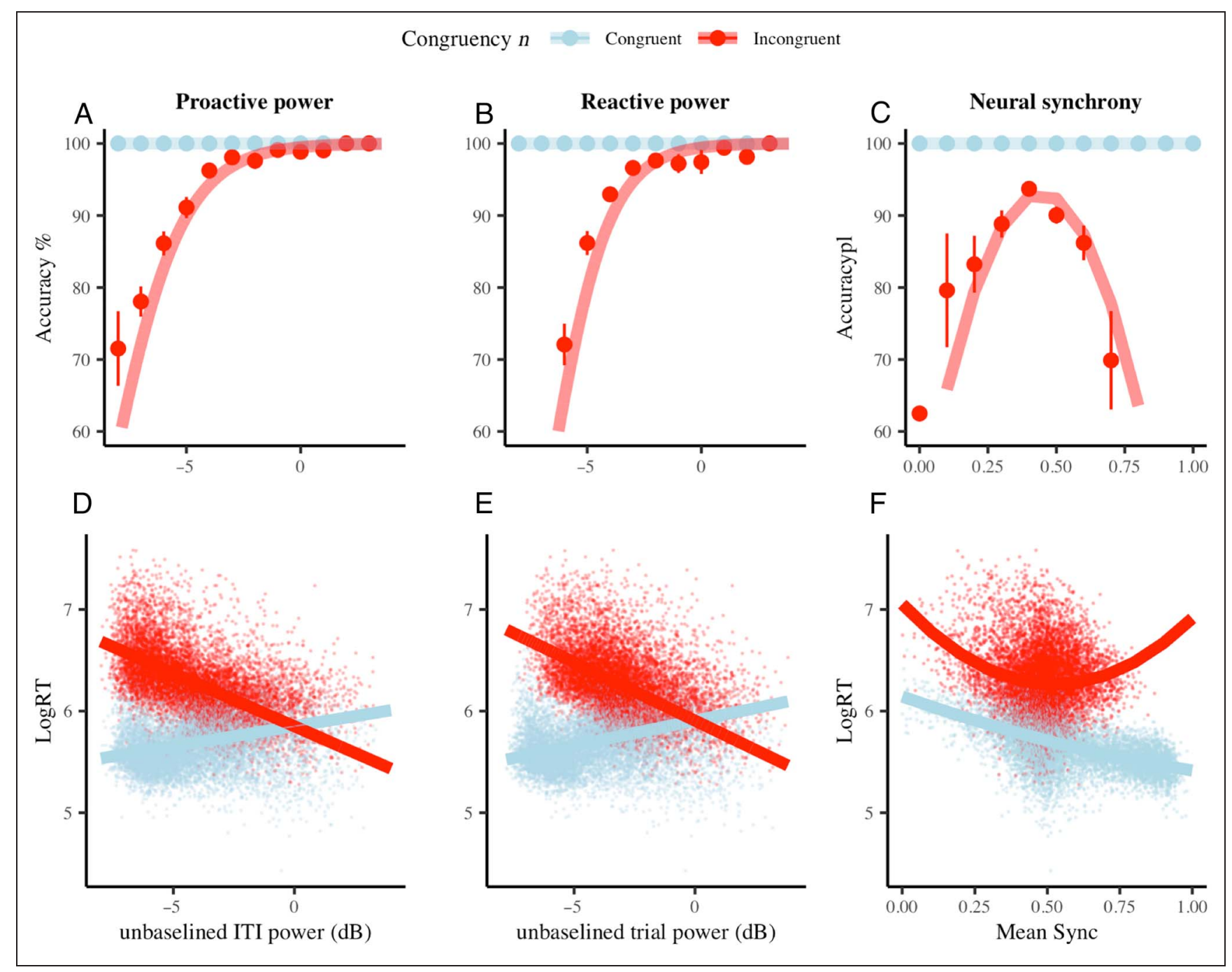

Figure 8. Relation between neural and behavioral data. Lines represent the fitted regression models. Dots represent the mean values for all trials and all simulations. Bars (in the accuracy plots) indicate 95\% confidence intervals. Because there are not as many trials for each value of power or synchrony, the width of the confidence intervals varies.

Hence, also here, there was a significant effect in accuracy $\left[\chi^{2}(1, n=30)=669, p<.001 ;\right.$ Figure $\left.7 \mathrm{C}\right]$ and RT $\left[\chi^{2}(1\right.$, $n=30)=311, p<.001$; Figure 8G]. Moreover, also the interaction between reactive control and congruency on trial $n$ reached significance for both accuracy $\left[\chi^{2}(1, n=\right.$ $30)=117, p<.001]$ and RT $\left[\chi^{2}(1, n=30)=24,458\right.$, $p<.001$ ]. These two effects (in reactive and proactive power) potentially explain the conflict-adaptation effect, but this hypothesis remains to be tested.

Neural synchrony was able to significantly predict both accuracy $\left[x^{2}(1, n=30)=16.95, p<.001\right.$; Figure 8D] and RT $\left[\mathrm{X}^{2}(1, n=30)=9104, p<.001\right.$; Figure $\left.8 \mathrm{H}\right]$. Moreover, also the interaction of synchrony with congruency on trial $n$ reached significance in RT $\left[\chi^{2}(1, n=30)=\right.$ $18,272, p<.001]$ and in accuracy $\left[\chi^{2}(1, n=30)=95\right.$, $p<.001]$. However, a linear model is clearly not appropriate for these data (see Figure $8 \mathrm{D}$ and $\mathrm{H}$ ). To remedy this, a mixed quadratic regression was fitted for both RT and accuracy. To guarantee model convergence, we did not implement the interaction of synchrony with congruency in this model but fitted the model for congruent and incongruent trials separately. For RT analyses, we found a significant effect of synchrony in congruent $\left[\chi^{2}(1, n=30)=465, p<.001\right]$ as well as incongruent $\left[\mathrm{X}^{2}(1, n=30)=369, p<.001\right]$ trials. In addition, also the quadratic effect of synchrony was significant in both congruent $\left[\chi^{2}(1, n=30)=51.62, p<\right.$ $.001]$ and incongruent $\left[\chi^{2}(1, n=30)=297, p<.001\right]$ trials. For the analyses of accuracy, only an intercept could be estimated in congruent trials, because accuracy was always $100 \%$ in this case. For incongruent trials, there was a significant linear $\left[\chi^{2}(1, n=30)=192, p<.001\right]$ and quadratic $\left[x^{2}(1, n=30)=217, p<.001\right]$ effect of synchrony. Hence, performance for incongruent trials was best at intermediate levels of synchrony. This is because, when synchrony was around 0.5 , only the relevant stimulus modules were synchronized with the response module. Instead, when synchrony is much lower, neither module is synchronized, and if synchrony is much higher, both modules are synchronized. In summary, whereas 
for incongruent trials, an intermediate level of synchrony (selective control) is optimal, congruent trials do benefit from higher levels of synchrony, as is observed by a faster RT at higher synchrony levels (Figure $8 \mathrm{H}$ ). This prediction also remains to be tested empirically.

\section{DISCUSSION}

Current work employed a computational modeling approach to gain insight in the underlying mechanisms that drive human adaptive cognitive control. For this purpose, we combined and extended previous modeling work on conflict monitoring (Botvinick et al., 2001) and neural synchrony (Verguts, 2017). Nine versions of the model were explored, which differed in the dimension (intensity or selectivity) or the time scale (slow or fast) (Braver, 2012; Scherbaum et al., 2012) for which control was adapted. Only the model that was adaptive on both the intensity and selectivity dimensions and on both time scales could account for human behavioral (Gratton et al., 1992) and neurophysiological (Pastötter et al., 2013) findings. In addition, this full adaptive model version provides predictions for empirical behavioral and neurophysiological data.

The current study relied on response level energy to evaluate the required intensity of control. By using this approach, the model ignored several features that might also play an important role in evaluating when to use control. In the current model, all information for control adaptation originates from internal (conflict) sources. However, control adaptation might also rely on other sources such as reward or contextual information in the environment. Learning from more sources of information would allow the model to better estimate the costs and benefits of control (Shenhav, Botvinick, \& Cohen, 2013), allowing control adaptation to be even more efficient.

For the selectivity dimension, the model also relied on energy as a measure of conflict. More specifically, as in previous work (Verguts \& Notebaert, 2008), we used a multiplication of bottom-up, top-down, and conflict signals to evaluate where control should be employed. In line with previous work (Melara \& Algom, 2003), it was argued that when conflict is low, an agent might benefit from additionally exploiting task-irrelevant but correlated information. Thus, when conflict is low, the model will distribute its control signal over the entire network (less selective). When conflict is high, the model will only send the control signal to the task-relevant dimension (more selective). Because low-conflict trials are characterized by the fact that task-irrelevant and task-relevant information provide evidence for the same response, evidence accumulation in the integrator unit of the model will be faster, leading to faster RT (see also Figure 2). Intriguingly, whereas the control signal in Botvinick et al. (2001) did not implement selectivity, we demonstrated that, in the sync model, adaptivity in the selectivity of control is a necessary requirement for reproducing the qualitative pattern of conflict-adaptation effects in behavior (performance benefit for congruent-congruent sequences). Such a perspective shifts theoretical attention toward consideration of all trial types (congruent, incongruent, neutral) and how they can be employed for optimal performance (Compton, Huber, Levinson, \& Zheutlin, 2012). Importantly, when multiple stimulus dimensions are added to the task, a selective adaptive model is required to also detect the source of conflict such that the agent can optimally select information from multiple nonconflicting dimensions. Future work should therefore also evaluate the costs and benefits of exploiting (nominally) task-irrelevant dimensions. This could be implemented by (reinforcement) learning the conflict-to-control weight matrix, the control-to-processing weight matrix (Verguts \& Notebaert, 2008), or the attention directed to specific dimensions (Roelfsema \& Van Ooyen, 2005; Kruschke, 1992, 2001). As demonstrated in previous work, the oscillatory model can easily be embedded in a general learning framework (Verbeke \& Verguts, 2019).

Importantly, when to use control was evaluated on two time scales. A weighted integration of conflict over the respective time scale (trials or time steps for slow and fast time scales, respectively) determined the level of control employed by the model. Although most models of control only implemented control on a slow time scale (but see Scherbaum et al., 2011), we show that implementing control on both time scales, simultaneously, allows the model to connect to previous neurophysiological findings that show a conflict-adaptation effect in midfrontal theta power (Pastötter et al., 2013). Moreover, implementing control adaptation on a fast time scale allows the model to deal with situations in which the relevant stimulus dimension needs to be inferred during the trial itself (stimulus-driven control; see also Bugg, 2012). The approach to use two time scales of control can be linked to previous theoretical (Braver, 2012) and empirical (e.g., Gonthier, Braver, \& Bugg, 2016; Ruge, Jamadar, Zimmermann, \& Karayanidis, 2013; Stuphorn \& Emeric, 2012) work that described a dual-mechanism framework for cognitive control. Here, control on a slow time scale is used proactively by synchronizing the network before trial onset, to prevent conflict to occur. Control on a fast time scale is used reactively during the trial itself, to eliminate all conflict that could not be prevented by proactive control. Note that, although in the current work proactive and reactive control could be differentiated by their respective time scales, the time scale of both types of control might depend on the task context (Jiang, Beck, Heller, \& Egner, 2015).

An important feature of the current model is that it accounts for the fact that neural activity is characterized by oscillations in different frequencies. Although, on a computational level, top-down biasing of lower-level processing can easily be simulated without oscillations (Shenhav et al., 2013; Botvinick et al., 2001; Verguts \& Notebaert, 2008; Cohen et al., 1990), a growing amount 
of empirical research indicates that the human brain uses synchrony to modulate processing efficiency between neural areas (Fries, 2005, 2015). Moreover, current work, as well as previous work (Verguts, 2017), illustrates that implementing oscillations allows a model to connect to a wider range of neurophysiological data. In addition, oscillatory processing might reveal important constraints (e.g., the need for adaptive selectivity) that are not applicable for other implementations of biasing signals. Future work should further explore the benefits and costs of oscillations for neural processing dynamics.

In the processing unit, oscillations were implemented at gamma frequency, in line with a wide range of empirical data that describe a critical role for gamma in cognitive processing (Lisman \& Jensen, 2013; Womelsdorf \& Fries, 2007; Gray \& Singer, 1989). In the MFC of the control unit, a theta frequency was used. This is consistent with numerous studies that link cognitive control, and more specifically control adaptation, to midfrontal theta power (van Driel et al., 2015; Cavanagh \& Frank, 2014). More specifically, the current model uses theta frequency in the MFC to send synchronizing bursts to gammafrequency oscillations in the processing unit. Importantly, as illustrated in the Appendix, model behavior can be reproduced by implementing other frequencies as well. Furthermore, previous work (Verbeke et al., 2021) has implemented theta frequency in the model processing unit as well. In this case, control and processing units communicate via within-frequency (theta-theta) instead of crossfrequency interactions (theta-gamma). Both types of interactions are supported by empirical EEG work (Verbeke et al., 2021; Siems \& Siegel, 2020; Szczepanski et al., 2014; Lisman \& Jensen, 2013; Cavanagh, Cohen, \& Allen, 2009; Jensen \& Colgin, 2007). Also in the control unit, different frequencies can lead to qualitatively similar results. However, previous work (Verbeke \& Verguts, 2019) was able to provide a more detailed insight in the importance of control frequency. Importantly, the controller frequency determines the frequency of bursts. When this frequency is slow, there is more time between bursts, which might allow oscillations in the processing unit to drift apart. Crucially, if the controller frequency is too fast, there may be too many bursts and not enough time where activity is actually oscillating. In such case, the BBS principle (Equation 5) would be negligible, which leads to a dramatic decrease in model performance, as was demonstrated in previous work (Verbeke \& Verguts, 2019). For simplicity, here, we employed the same control frequency for all trials. However, as illustrated in recent work, frequency might also change as a function of task difficulty (Senoussi et al., 2021). In summary, an important avenue for future work is to further explore the computational role of different frequency bands.

Current work has demonstrated how implementing oscillatory processing allows an improved insight into the mechanisms underlying cognitive processes. For example, it allows the model to connect to a wider range of empirical data (Schmidt \& Weissman, 2014; Pastötter et al., 2013; Gratton et al., 1992) and provide testable predictions for future empirical work. For instance, we hypothesize an inverse conflict-adaptation effect in synchrony/phase coupling between task-related input (e.g., visual) and output (e.g., motor) areas. Note that this hypothesis considers more posterior task-related areas and hence crucially differs from previous findings showing an increase of phase coupling between frontal areas during incongruent trials (Hanslmayr et al., 2008). Importantly, we collapsed our measure of synchrony over the full input (color and word dimensions) or output (response dimension) layers of the model. As a result, our hypotheses are testable with neurophysiological measurements. In addition, the model hypothesizes that both synchrony and midfrontal theta power can predict accuracy and RTs on a trial-to-trial basis.

In the current model, cognitive control is established through a cooperation between the MFC and LFC. We propose that the MFC determines the intensity of control. More specifically, stronger theta power in the MFC results in an increase of synchronizing bursts that are sent to lower-level processing areas (e.g., posterior visual or motor areas). Given its involvement in both proactive and reactive cognitive control (Stuphorn \& Emeric, 2012; Miller \& Cohen, 2001) and the strong anatomical connectivity between MFC and posterior processing and motor control areas (Silvetti, Alexander, Verguts, \& Brown, 2014), MFC seems optimally suited for sending these bursts. We propose that selecting where to send the bursts is anatomically linked to the LFC. This is consistent with the notion of (dorso)LFC as a holder of task demands (Mac Donald, Cohen, Stenger, \& Carter, 2000; Braver et al., 1997). Both LFC and MFC receive information from the conflict monitoring unit, on which basis they decide the required intensity and selectivity of control. Previous work also linked this conflict monitoring unit to MFC (Botvinick, Cohen, \& Carter, 2004).

In summary, the current work has described an oscillatory model of adaptive cognitive control. The model implements cognitive control on two time scales, by top-down modulated gating of neural activity via synchronization. It provides new insights into adaptive cognitive control and opens avenues to further explore how learning modulates cognitive control. Moreover, the model simulations were consistent with previous behavioral and neurophysiological data and provided predictions for future empirical work.

\section{APPENDIX: PARAMETER EXPLORATION}

\section{Methods}

To gain insight in the parameter space of the model, three grid searches were performed. In the first parameter exploration, we focused on parameters in the conflict monitoring and control unit. Here, we explored from Equation 8 


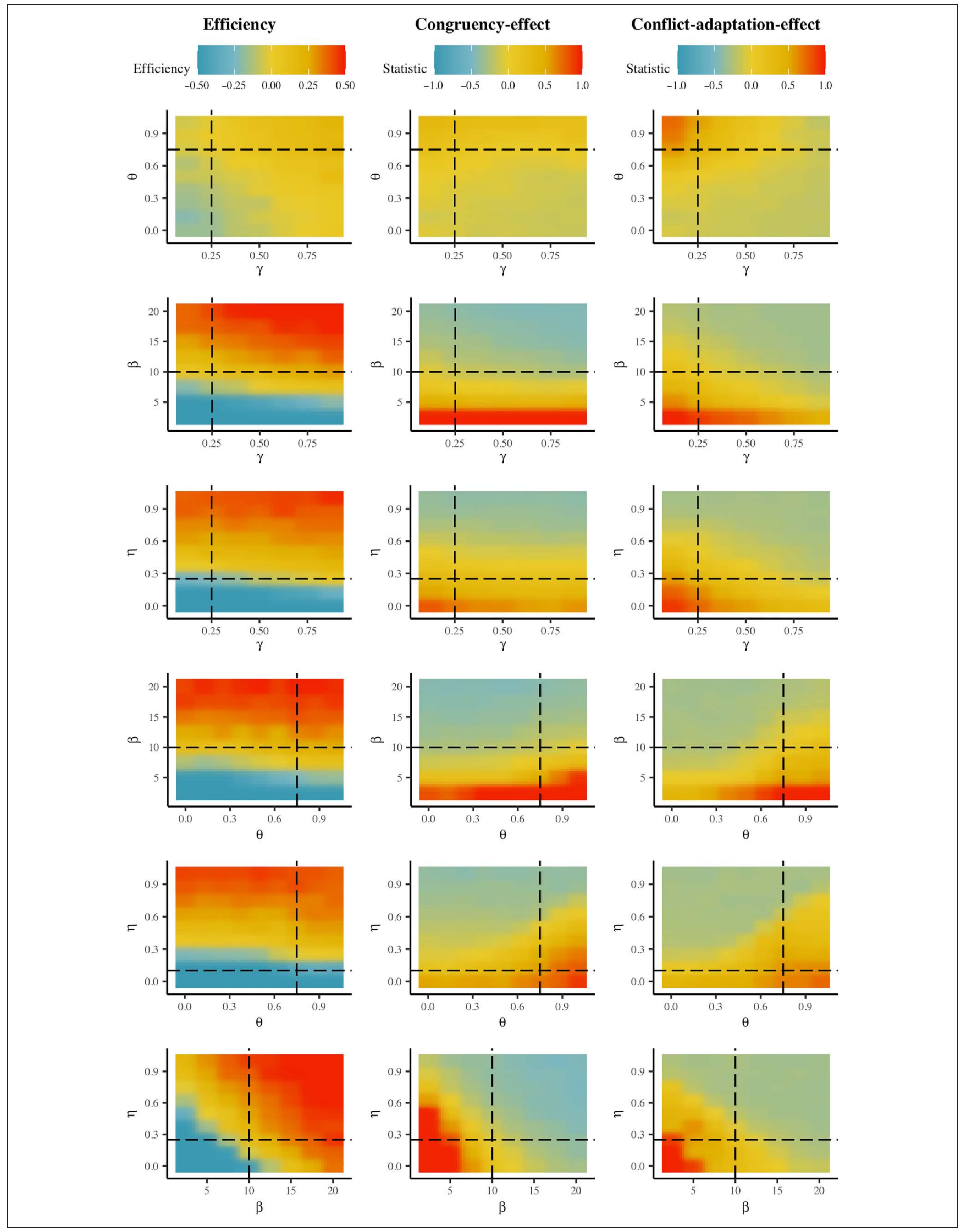

Figure A1. Parameter exploration for the conflict monitoring unit. Each panel presents results for one pair of parameters. Here, statistics and efficiency are averaged across all values for the not-shown parameters. Black dashed lines indicate parameter values that were used to simulate the full adaptive model in the main text. 
the $\gamma_{\text {proactive, }} \eta_{\text {proactive }}$, and $\beta$ parameters. In addition, we explored different values for the $\theta_{\text {selective }}$ parameter from Equation 11. More specifically, for $\gamma_{\text {proactive }}$, values ranged from 0.125 to 0.875 in steps of 0.125 ; for $\eta_{\text {proactive, we ex- }}$ plore values from 0 to 1 in steps of $0.125 ; \beta$ was evaluated for values ranging from 2.5 to 20 in steps of 2.5 ; and $\theta_{\text {selective }}$ took on values from 0 to 1 in steps of 0.125 . All other parameter values were set as described for the full adaptive model from the main text. We performed one simulation of 600 trials for each parameter combination.

In the second grid search, we focused on the integrator unit. Here, we explored the lateral inhibition implemented by the off-diagonal elements of $L$ (see Equation 6 ), the response threshold, and the standard deviation of the noise $(n)$. For "inhibition" (off-diagonal elements of $L$ ), we explored values from 0 to 0.1 , with a step size of 0.005 . As for threshold, we searched values from 5 to 40 with a step size of 5 . For noise, we looped over values from 0 to 0.5 with a step size of 0.05 . Again, all other parameter values were set as described for the full adaptive model from the main text. We performed one simulation of 600 trials for each parameter combination.

The third grid search focused on the frequencies of the phase code neurons that were used in the control and processing unit. Here, we explored control frequencies ranging from 2 to $20 \mathrm{~Hz}$ in steps of $2 \mathrm{~Hz}$ and processing frequencies ranging from 10 to $100 \mathrm{~Hz}$ in steps of $10 \mathrm{~Hz}$. Note that, in the processing unit, there was always a $2.5 \%$ difference in frequency between the stimulus and response nodes. Also here, all other parameter values were set as described for the full adaptive model from the main text. We performed one simulation of 600 trials for each parameter combination.

\section{Analyses}

Both RT and accuracy data from each simulation were analyzed. Because there was only one simulation for each parameter combination, the random intercept was removed from the linear mixed models that we used to analyze simulations in the main text, resulting in a linear model for RT and generalized linear model for accuracy. Again, congruency on trial $n$ and congruency on trial $n-1$ were used as independent variables. For illustrative purposes, the accuracy and RT results of parameter explorations were combined. Here, we defined the mean of the mean of the $z$-scored accuracy and negative $z$-scored RT as a measure of efficiency. For measures of the congruency
Figure A2. Parameter exploration for the integrator unit. Each panel presents results for one pair of parameters. Here, statistics and efficiency are averaged across all values for the not-shown parameter. Black dashed lines indicate parameter values that were used to simulate the full adaptive model in the main text.

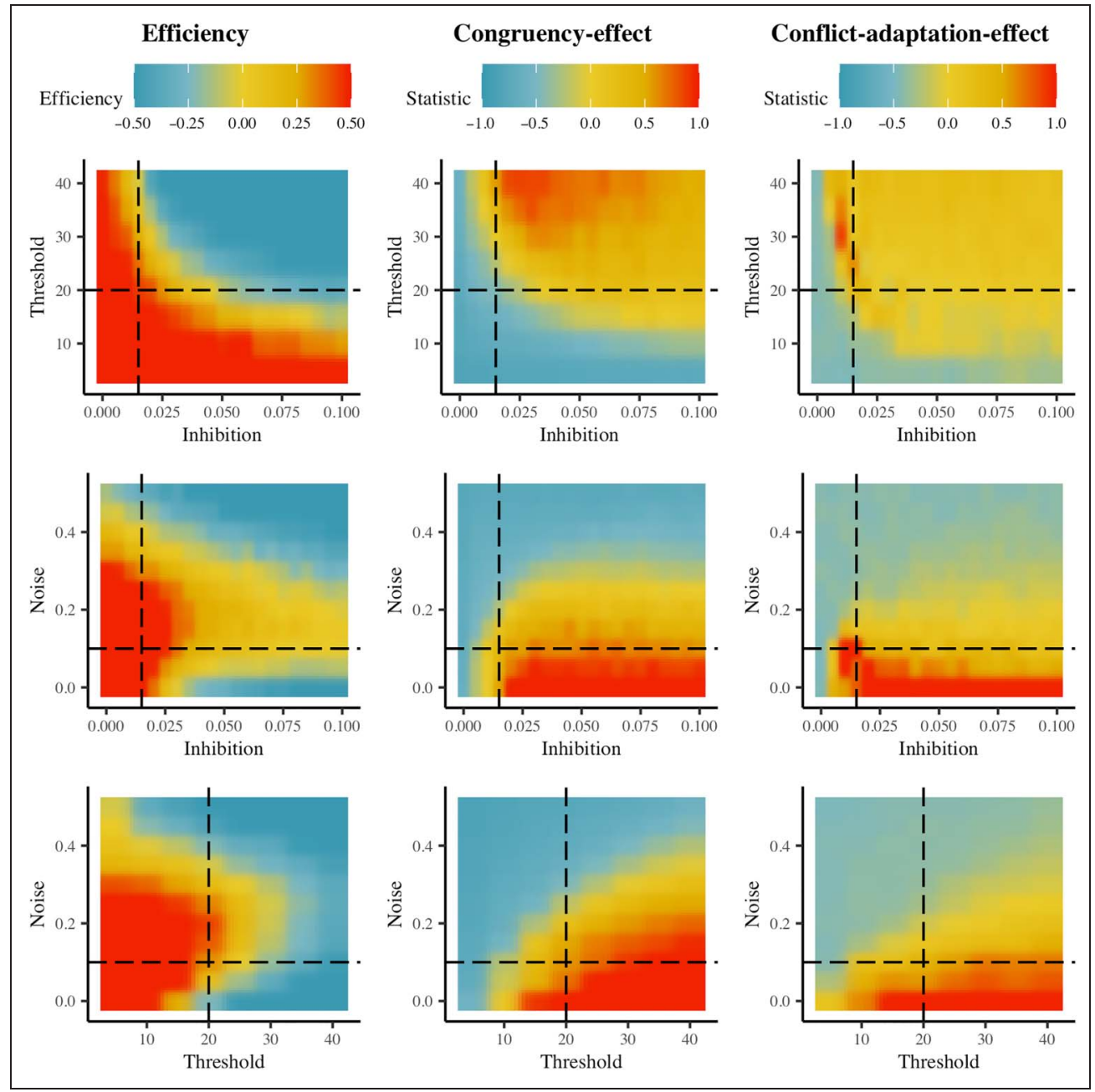


Figure A3. Parameter exploration for frequencies in the control and processing unit. Each panel presents results for one pair of parameters. Black dashed lines indicate parameter values that were used to simulate the full adaptive model in the main text.

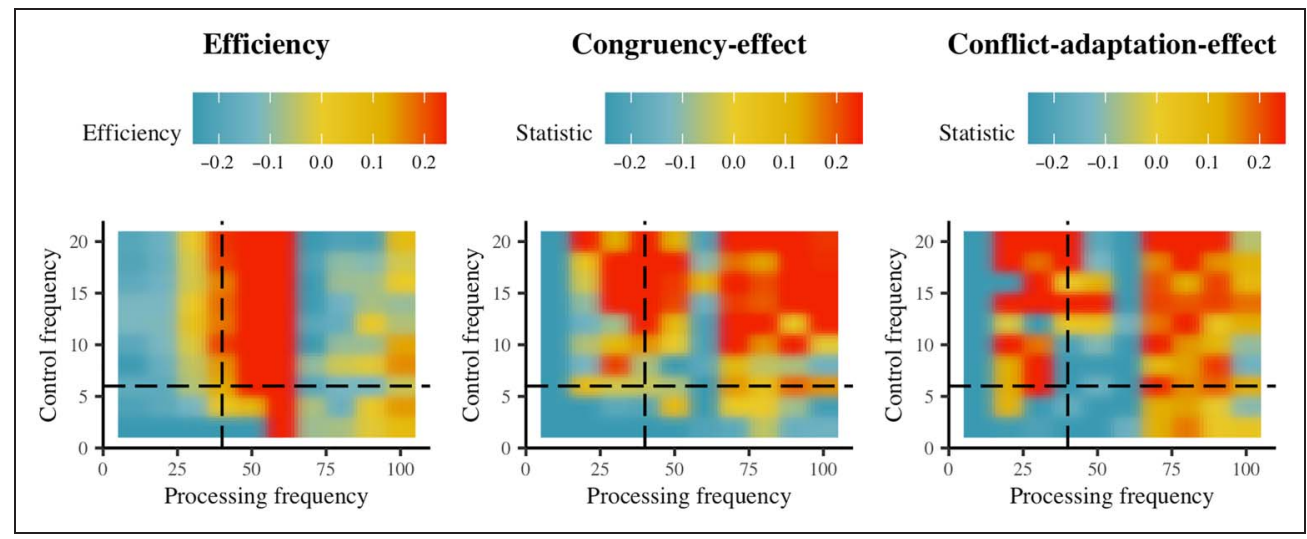

and conflict-adaptation effect, we used the $z$-scored statistic that resulted from the linear ( $F$ value) or generalized linear model ( $x^{2}$ value).

\section{Results}

Results from the parameter explorations are presented in Figure A1-A3. Here, we present heat maps for efficiency (combination of RT and accuracy; see Methods), the congruency effect (on trial $n$ ), and the conflict-adaptation effect (interaction of congruency on trial $n$ with congruency on $n-1$ ). Figure A1 presents results of the conflict monitoring and control unit exploration. Figure A2 presents results of the integrator unit exploration, and Figure $\mathrm{A} 3$ presents results of the frequency exploration in the control and processing unit.

Reprint requests should be sent to Pieter Verbeke, Department of Experimental Psychology, Ghent University, H. Dunantlaan 2, Ghent, Belgium 9000, or via e-mail: pjverbek.verbeke@ugent.be.

\section{Data Availability}

Code to simulate the model and analyze the resulting data is provided in our GitHub repository: github.com /CogComNeuroSci/PieterV_public/tree/master/conflict _synchrony.

\section{Funding Information}

Pieter Verbeke: Research Foundation Flanders (Fonds Wetenschappelijk Onderzoek; https://dx.doi.org/10 .13039/501100003130), grant number: 1102519 N. Tom Verguts: Ghent University Research Council, grant number: BOF17/GOA/004.

\section{Diversity in Citation Practices}

A retrospective analysis of the citations in every article published in this journal from 2010 to 2020 has revealed a persistent pattern of gender imbalance: Although the proportions of authorship teams (categorized by estimated gender identification of first author/last author) publishing in the Journal of Cognitive Neuroscience (JoCN) during this period were $\mathrm{M}(\mathrm{an}) / \mathrm{M}=.408, \mathrm{~W}(\mathrm{oman}) / \mathrm{M}=.335$, $\mathrm{M} / \mathrm{W}=.108$, and $\mathrm{W} / \mathrm{W}=.149$, the comparable proportions for the articles that these authorship teams cited were $\mathrm{M} / \mathrm{M}=.579, \mathrm{~W} / \mathrm{M}=.243, \mathrm{M} / \mathrm{W}=.102$, and $\mathrm{W} / \mathrm{W}=.076$ (Fulvio et al., JoCN, 33:1, pp. 3-7). Consequently, JoCN encourages all authors to consider gender balance explicitly when selecting which articles to cite and gives them the opportunity to report their article's gender citation balance. The authors of this article report its proportions of citations by gender category to be as follows: $\mathrm{M} / \mathrm{M}=$ $.833, \mathrm{~W} / \mathrm{M}=.024, \mathrm{M} / \mathrm{W}=.095$, and $\mathrm{W} / \mathrm{W}=.048$.

\section{REFERENCES}

Botvinick, M. M., Braver, T. S., Barch, D. M., Carter, C. S., \& Cohen, J. D. (2001). Conflict monitoring and cognitive control. Psychological Review, 108, 624-652. https://doi.org /10.1037/0033-295X.108.3.624, PubMed: 11488380

Botvinick, M. M., Cohen, J. D., \& Carter, C. S. (2004). Conflict monitoring and anterior cingulate cortex: An update. Trends in Cognitive Sciences, 8, 539-546. https://doi.org/10.1016/j .tics.2004.10.003, PubMed: 15556023

Braem, S., Bugg, J. M., Schmidt, J. R., Crump, M. J. C., Weissman, D. H., Notebaert, W., et al. (2019). Measuring adaptive control in conflict tasks. Trends in Cognitive Sciences, 23, 769-783. https://doi.org/10.1016/j.tics.2019.07.002, PubMed: 31331794

Braver, T. S. (2012). The variable nature of cognitive control: A dual mechanisms framework. Trends in Cognitive Sciences, 16, 106-113. https://doi.org/10.1016/j.tics.2011.12.010, PubMed: 22245618

Braver, T. S., Cohen, J. D., Nystrom, L. E., Jonides, J., Smith, E. E., \& Noll, D. C. (1997). A parametric study of prefrontal cortex involvement in human working memory. Neuroimage, 5 , 49-62. https://doi.org/10.1006/nimg.1996.0247, PubMed: 9038284

Bugg, J. M. (2012). Dissociating levels of cognitive control: The case of Stroop interference. Current Directions in Psychological Science, 21, 302-309. https://doi.org/10.1177/0963721412453586

Cavanagh, J. F., Cohen, M. X., \& Allen, J. J. B. (2009). Prelude to and resolution of an error: EEG phase synchrony reveals cognitive control dynamics during action monitoring. Journal of Neuroscience, 29, 98-105. https://doi.org/10.1523 /JNEUROSCI.4137-08.2009, PubMed: 19129388

Cavanagh, J. F., \& Frank, M. J. (2014). Frontal theta as a mechanism for cognitive control. Trends in Cognitive 
Sciences, 18, 414-421. https://doi.org/10.1016/j.tics.2014.04 .012, PubMed: 24835663

Cavanagh, J. F., Frank, M. J., Klein, T. J., \& Allen, J. J. B. (2010). Frontal theta links prediction errors to behavioral adaptation in reinforcement learning. Neuroimage, 49, 3198-3209. https://doi.org/10.1016/j.neuroimage.2009.11.080, PubMed: 19969093

Cohen, J. D., Dunbar, K., \& McClelland, J. L. (1990). On the control of automatic processes: A parallel distributed processing account of the Stroop effect. Psychological Review, 97, 332-361. https://doi.org/10.1037/0033-295X.97.3 .332, PubMed: 2200075

Compton, R. J., Huber, E., Levinson, A. R., \& Zheutlin, A. (2012). Is "conflict adaptation" driven by conflict? Behavioral and EEG evidence for the underappreciated role of congruent trials. Psychophysiology, 49, 583-589. https://doi.org/10.1111 j.1469-8986.2012.01354.x, PubMed: 22332754

Egner, T. (2007). Congruency sequence effects and cognitive control. Cognitive, Affective \& Behavioral Neuroscience, 7 , 380-390. https://doi.org/10.3758/CABN.7.4.380, PubMed: 18189011

Engel, A. K., Fries, P., \& Singer, W. (2001). Dynamic predictions: Oscillations and synchrony in top-down processing. Nature Reviews Neuroscience, 2, 704-716. https://doi.org/10.1038 /35094565, PubMed: 11584308

Fries, P. (2005). A mechanism for cognitive dynamics: Neuronal communication through neuronal coherence. Trends in Cognitive Sciences, 9, 474-480. https://doi.org/10.1016/j.tics 2005.08.011, PubMed: 16150631

Fries, P. (2015). Rhythms for cognition: Communication through coherence. Neuron, 88, 220-235. https://doi.org/10 .1016/j.neuron.2015.09.034, PubMed: 26447583

Gonthier, C., Braver, T. S., \& Bugg, J. M. (2016). Dissociating proactive and reactive control in the Stroop task. Memory and Cognition, 44, 778-788. https://doi.org/10.3758/s13421 -016-0591-1, PubMed: 26861210

Gratton, G., Coles, M. G. H., \& Donchin, E. (1992). Optimizing the use of information: Strategic control of activation of responses. Journal of Experimental Psychology: General, 121, 480-506. https://doi.org/10.1037/0096-3445.121.4.480, PubMed: 1431740

Gray, C. M., \& Singer, W. (1989). Stimulus-specific neuronal oscillations in orientation columns of cat visual cortex. Proceedings of the National Academy of Sciences, U.S.A., 86 1698-1702. https://doi.org/10.1073/pnas.86.5.1698, PubMed: 2922407

Hanslmayr, S., Pastötter, B., Bäuml, K. H., Gruber, S., Wimber, M., \& Klimesch, W. (2008). The electrophysiological dynamics of interference during the stroop task. Journal of Cognitive Neuroscience, 20, 215-225. https://doi.org/10.1162/jocn.2008 .20020, PubMed: 18275330

Helfrich, R. F., \& Knight, R. T. (2016). Oscillatory dynamics of prefrontal cognitive control. Trends in Cognitive Sciences, 20, 916-930. https://doi.org/10.1016/j.tics.2016.09.007, PubMed: 27743685

Jensen, O., \& Colgin, L. L. (2007). Cross-frequency coupling between neuronal oscillations. Trends in Cognitive Sciences, 11, 267-269. https://doi.org/10.1016/j.tics.2007.05.003, PubMed: 17548233

Jiang, J., Beck, J., Heller, K., \& Egner, T. (2015). An insulafrontostriatal network mediates flexible cognitive control by adaptively predicting changing control demands. Nature Communications, 6, 8165. https://doi.org/10.1038 /ncomms9165, PubMed: 26391305

Kruschke, J. K. (1992). ALCOVE: An exemplar-based connectionist model of category learning. Psychological Review, 99, 22-44. https://doi.org/10.1037/0033-295X.99.1 .22, PubMed: 1546117
Kruschke, J. K. (2001). Toward a unified model of attention in associative learning. Journal of Mathematical Psychology, 45, 812-863. https://doi.org/10.1006/jmps.2000.1354

Lisman, J. E., \& Jensen, O. (2013). The theta-gamma neural code. Neuron, 77, 1002-1016. https://doi.org/10.1016/j .neuron.2013.03.007, PubMed: 23522038

Mac Donald, A. W., Cohen, J. D., Stenger, A. V., \& Carter, C. S. (2000). Dissociating the role of the dorsolateral prefrontal and anterior cingulate cortex in cognitive control. Science, 288, 1835-1838. https://doi.org/10.1126/science.288.5472 .1835, PubMed: 10846167

Melara, R. D., \& Algom, D. (2003). Driven by information: A tectonic theory of stroop effects. Psychological Review, 110, 422-471. https://doi.org/10.1037/0033-295X.110.3.422, PubMed: 12885110

Miller, E. K., \& Cohen, J. D. (2001). An integrative theory of prefrontal cortex function. Annual Review of Neuroscience, 24, 167-202. https://doi.org/10.1146/annurev.neuro.24.1.167, PubMed: 11283309

Nigbur, R., Cohen, M. X., Ridderinkhof, K. R., \& Stürmer, B. (2011). Theta dynamics reveal domain-specific control over stimulus and response conflict. Journal of Cognitive Neuroscience, 24, 1264-1274. https://doi.org/10.1162/jocn_a 00128, PubMed: 21861681

Norman, D. A., \& Shallice, T. (1986). Attention to action: Willed and automatic control of behavior. In R. J. Davidson, G. E. Schwartz, \& D. Shapiro (Eds.), Consciousness and selfregulation (pp. 1-18). Boston: Springer. https://doi.org/10 .1007/978-1-4757-0629-1_1

Pastötter, B., Dreisbach, G., \& Bäuml, K. H. T. (2013). Dynamic adjustments of cognitive control: Oscillatory correlates of the conflict adaptation effect. Journal of Cognitive Neuroscience, 25, 2167-2178. https://doi.org/10.1162/jocn_a_00474, PubMed: 24001006

Roelfsema, P. R., \& Van Ooyen, A. (2005). Attention-gated reinforcement learning of internal representations for classification. Neural Computation, 17, 2176-2214. https:// doi.org/10.1162/0899766054615699, PubMed: 16105222

Ruge, H., Jamadar, S., Zimmermann, U., \& Karayanidis, F. (2013). The many faces of preparatory control in task switching: Reviewing a decade of fMRI research. Human Brain Mapping, 34, 12-35. https://doi.org/10.1002/hbm 21420, PubMed: 21998090

Scherbaum, S., Dshemuchadse, M., Ruge, H., \& Goschke, T (2012). Dynamic goal states: Adjusting cognitive control without conflict monitoring. Neuroimage, 63, 126-136. https://doi.org/10.1016/j.neuroimage.2012.06.021, PubMed: 22732559

Scherbaum, S., Fischer, R., Dshemuchadse, M., \& Goschke, T. (2011). The dynamics of cognitive control: Evidence for within-trial conflict adaptation from frequency-tagged EEG. Psychophysiology, 48, 591-600. https://doi.org/10.1111/j.1469 -8986.2010.01137.x, PubMed: 21044093

Schmidt, J. R., \& Weissman, D. H. (2014). Congruency sequence effects without feature integration or contingency learning confounds. PLoS One, 9, 1-9. https://doi.org/10.1371 /journal.pone.0102337, PubMed: 25019526

Senoussi, M., Verbeke, P., Desender, K., De Loof, E., Talsma, D., \& Verguts, T. (2021). Theta oscillations shift towards optimal frequency for cognitive control. BioRxiv , 1-20. https://doi.org $/ 10.1101 / 2020.08 .30 .273706$

Shenhav, A., Botvinick, M. M., \& Cohen, J. D. (2013). The expected value of control: An integrative theory of anterior cingulate cortex function. Neuron, 79, 217-240. https://doi.org/10.1016/j.neuron.2013.07.007, PubMed: 23889930

Siems, M., \& Siegel, M. (2020). Dissociated neuronal phaseand amplitude-coupling patterns in the human brain. 
Neuroimage, 209, 116538. https://doi.org/10.1016/j .neuroimage.2020.116538, PubMed: 31935522

Silvetti, M., Alexander, W., Verguts, T., \& Brown, J. W. (2014). From conflict management to reward-based decision making: Actors and critics in primate medial frontal cortex. Neuroscience \& Biobehavioral Reviews, 46, 44-57. https:// doi.org/10.1016/j.neubiorev.2013.11.003, PubMed: 24239852

Springer, M., \& Paulsson, J. (2006). Harmonies from noise. Nature, 439, 27-29. https://doi.org/doi:10.1038/439027a, PubMed: 16397487

Stuphorn, V., \& Emeric, E. E. (2012). Proactive and reactive control by the medial frontal cortex. Frontiers in Neuroengineering, 5, 1-11. https://doi.org/10.3389/fneng .2012.00009, PubMed: 22723779

Szczepanski, S. M., Crone, N. E., Kuperman, R. A., Auguste, K. I., Parvizi, J., \& Knight, R. T. (2014). Dynamic changes in phaseamplitude coupling facilitate spatial attention control in fronto-parietal cortex. PLoS Biology, 12, e1001936. https://doi .org/10.1371/journal.pbio.1001936, PubMed: 25157678

Usher, M., \& McClelland, J. L. (2001). The time course of perceptual choice: The leaky, competing accumulator model. Psychological Review, 108, 550-592. https://doi.org/10.1037 10033-295X.108.3.550, PubMed: 11488378

van den Berg, R., Awh, E., \& Ma, W. J. (2012). Factorial comparison of working memory models ronald. Psychological Review, 121, 124-149. https://doi.org/10.1037 /a0035234, PubMed: 24490791

van Driel, J., Swart, J. C., Egner, T., Ridderinkhof, K. R., \& Cohen, M. X. (2015). (No) time for control: Frontal theta dynamics reveal the cost of temporally guided conflict anticipation. Cognitive, Affective \& Behavioral Neuroscience, 15, 787-807. https://doi.org/10.3758/s13415-015-0367-2, PubMed: 26111755

Verbeke, P., Ergo, K., De Loof, E., Verguts, T., De Loof, E., \& Verguts, T. (2021). Learning to synchronize: Midfrontal theta dynamics during rule switching. Journal of Neuroscience, 41 1-13. https://doi.org/10.1523/JNEUROSCI.1874-20.2020, PubMed: 33310756

Verbeke, P., \& Verguts, T. (2019). Learning to synchronize: How biological agents can couple neural task modules for dealing with the stability-plasticity dilemma. PLoS Computational Biology, 15, e1006604. https://doi.org/10.1371/journal.pcbi .1006604, PubMed: 31430280

Verguts, T. (2017). Binding by random bursts: A computational model of cognitive control. Journal of Cognitive

Neuroscience, 29, 1103-1118. https://doi.org/10.1162/jocn_a 01117, PubMed: 28253078

Verguts, T., \& Notebaert, W. (2008). Hebbian learning of cognitive control: Dealing with specific and nonspecific adaptation. Psychological Review, 115, 518-525. https://doi .org/10.1037/0033-295X.115.2.518, PubMed: 18426302

Voloh, B., Valiante, T. A., Everling, S., \& Womelsdorf, T. (2015). Theta-gamma coordination between anterior cingulate and prefrontal cortex indexes correct attention shifts. Proceedings of the National Academy of Sciences, U.S.A., 112, 8457-8462. https://doi.org/10.1073/pnas.1500438112, PubMed: 26100868

Voytek, B., Kayser, A. S., Badre, D., Fegen, D., Chang, E. F., Crone, N. E., et al. (2015). Oscillatory dynamics coordinating human frontal networks in support of goal maintenance. Nature Neuroscience, 18, 1318-1324. https://doi.org/10.1038 /nn.4071, PubMed: 26214371

Womelsdorf, T., \& Fries, P. (2007). The role of neuronal synchronization in selective attention. Current Opinion in Neurobiology, 17, 154-160. https://doi.org/10.1016/j.conb 2007.02.002, PubMed: 17306527

Zhou, T., Chen, L., \& Aihara, K. (2005). Molecular communication through stochastic synchronization induced by extracellular fluctuations. Physical Review Letters, 95, 2-5. https://doi.org/10 .1103/PhysRevLett.95.178103, PubMed: 16383875 